\title{
Gamma-ray line from electroweakly interacting non-abelian spin-1 dark matter
}

\author{
Tomohiro Abe, ${ }^{a}$ Motoko Fujiwara, ${ }^{b}$ Junji Hisano $^{c, b, d}$ and Kohei Matsushita ${ }^{b}$ \\ ${ }^{a}$ Department of Physics, Faculty of Science and Technology, Tokyo University of Science, \\ 2641 Yamazaki, Noda, Chiba 278-8510, Japan \\ ${ }^{b}$ Department of Physics, Nagoya University, \\ Furo-cho Chikusa-ku, Nagoya, 464-8602 Japan \\ ${ }^{c}$ Kobayashi-Maskawa Institute for the Origin of Particles and the Universe, Nagoya University, \\ Furo-cho Chikusa-ku, Nagoya, 464-8602 Japan \\ ${ }^{d}$ Kavli IPMU (WPI), UTIAS, University of Tokyo, \\ Kashiwa, 277-8584, Japan \\ E-mail: abe.tomohiro@rs.tus.ac.jp, motoko@eken.phys.nagoya-u.ac.jp, \\ hisano@eken.phys.nagoya-u.ac.jp, kohei@eken.phys.nagoya-u.ac.jp
}

ABSTRACT: We study gamma-ray line signatures from electroweakly interacting nonabelian spin-1 dark matter (DM). In this model, $Z_{2}$-odd spin-1 particles including a DM candidate have the $\mathrm{SU}(2)_{L}$ triplet-like features, and the Sommerfeld enhancement is relevant in the annihilation processes. We derive the annihilation cross sections contributing to the photon emission and compare with the $\mathrm{SU}(2)_{L}$ triplet fermions, such as Wino DM in the supersymmetric Standard Model. The Sommerfeld enhancement factor is approximately the same in both systems, while our spin-1 DM predicts the larger annihilation cross sections into $\gamma \gamma / Z \gamma$ modes than those of the Wino by $\frac{38}{9}$. This is because a spin-1 DM pair forms not only $J=0$ but also $J=2$ partial wave states where $J$ denotes the total spin angular momentum. Our spin-1 DM also has a new annihilation mode into $Z_{2}$-even extra heavy vector and photon, $Z^{\prime} \gamma$. For this mode, the photon energy depends on the masses of DM and the heavy vector, and thus we have a chance to probe the mass spectrum. The latest gamma-ray line search in the Galactic Center region gives a strong constraint on our spin-1 DM. We can probe the DM mass for $\lesssim 25.3 \mathrm{TeV}$ by the Cherenkov Telescope Array experiment even if we assume a conservative DM density profile.

Keywords: Beyond Standard Model, Cosmology of Theories beyond the SM, Effective Field Theories

ArXiv EPrint: 2107.10029 


\section{Contents}

1 Introduction 1

2 Model 2

2.1 Physical spectrum 5

2.2 Couplings of $V$-particles 8

3 Annihilation cross section for $\boldsymbol{V}$-particles $\quad 8$

3.1 Non-relativistic field operators for $V$-particles 9

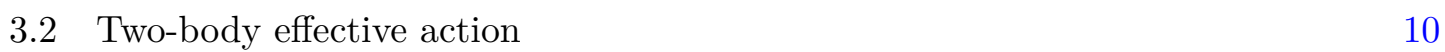

$\begin{array}{ll}3.3 & \text { Annihilation cross section } \\ \end{array}$

4 Gamma-ray line signatures $\quad 13$

$\begin{array}{lll}4.1 & \text { Line cross section } & 14\end{array}$

$\begin{array}{lll}4.2 & \text { Constraint from gamma-ray line signatures } & 15\end{array}$

5 Conclusions $\quad 19$

$\begin{array}{lr}\text { A Derivation of effective action } & 20\end{array}$

A.1 Real part of potential 20

A.2 Imaginary part of potential $\quad 21$

$\begin{array}{ll}\text { A.2.1 Matching procedure } & 21\end{array}$

A.2.2 Derivation of $\hat{\Gamma}_{\gamma \gamma}^{J} \quad 22$

\section{Introduction}

We have overwhelming evidence that suggests dark matter (DM) in our universe. Although the nature of DM remains unrevealed, we have one quantitative piece of information about DM, the DM energy density in the current universe. This energy density is determined to be $\Omega h^{2}=0.120 \pm 0.001$ by the Planck collaboration assuming the $\Lambda$ CDM cosmological model [1].

One of the most promising DM candidates is the Weakly Interacting Massive Particles (WIMPs). If we assume the WIMPs in our expanding universe, we can explain the current DM energy density as the thermal relic abundance through the Freeze-out mechanism [2]. In this scenario, the velocity-weighted annihilation cross section for DM, $\left\langle\sigma v_{\text {rel }}\right\rangle$, characterizes the prediction of DM abundance. To reach the observed DM energy density, we need the value of the canonical cross section, $\left\langle\sigma v_{\text {rel }}\right\rangle \simeq 3 \times 10^{-26} \mathrm{~cm}^{3} / \mathrm{s}$. This value is obtained once we assume DM mass and couplings to be the typical values of those in the electroweak theory in the Standard Model (SM). This fact is our motivation to consider DM candidates with electroweak interactions. 
Spin-0 and spin-1/2 DM with electroweak interactions are systematically studied in the contexts of the Minimal DM [3-5]. ${ }^{1}$ The Minimal Supersymmetric SM predicts concrete DM candidates stabilized by the symmetry called R-parity, which is studied in many contexts [7-10]. The model of spin-1 DM with electroweak interactions is also studied in the extra-dimensional models [11-14].

To reveal the general features of spin-1 DM, we construct the renormalizable fourdimensional model of electroweakly interacting non-abelian spin-1 DM [15]. This model is the minimal setup that realizes the fundamental features of the five-dimensional model of the spin- 1 DM such as the $Z_{2}$-parity and the degenerated mass spectrum. We impose the exchange symmetry between the gauge groups to realize the above features, which is inspired by the method of deconstructing dimensions [16, 17]. Our spin-1 DM couples to the electroweak gauge bosons with the electroweak gauge couplings, and the DM mass should be $\gtrsim \mathcal{O}(1) \mathrm{TeV}$ to explain the correct DM energy density. In this region, the electroweak bosons form the approximately long-range force potential. Consequently, the Sommerfeld enhancement is relevant in the DM annihilation processes as discussed in the other electroweakly interacting DM models [18-23]. Due to this enhancement, monochromatic spectral gamma-ray lines from the DM annihilation are the striking signals for our spin-1 DM.

In this paper, we study gamma-ray line signatures from electroweakly interacting nonabelian spin-1 DM. We make a comparison with the pure Wino DM, which is the spin- $\frac{1}{2}$ $\mathrm{DM}$ candidate of the $\mathrm{SU}(2)_{L}$ triplet in supersymmetric models, and clarify the differences between these systems. We derive the constraint from the latest gamma-ray observation in the Galactic Center region. We also reveal the region where we can probe in the future gamma-ray observation.

The rest of the paper is organized as follows. In section 2, we briefly review the electroweakly interacting non-abelian spin-1 DM. In section 3, we show the two-body effective action for the spin-1 DM system. We also show the annihilation cross section contributing to the monochromatic gamma-ray signals. In section 4, we present our numerical results. We compare the predicted annihilation cross section with that of the Wino DM and figure out distinctive features of our spin-1 DM. We show the constraints from the latest search for a monochromatic spectral line and the prospect sensitivity in the future gamma-ray observation. Our conclusions are given in section 5. We show the derivation of the two-body effective action in appendix A.

\section{Model}

We briefly introduce a model of electroweakly interacting non-abelian vector DM [15]. We extend the electroweak gauge symmetry in the $\mathrm{SM}$ into $\mathrm{SU}(2)_{0} \times \mathrm{SU}(2)_{1} \times \mathrm{SU}(2)_{2} \times \mathrm{U}(1)_{Y}$. The gauge bosons for $\mathrm{SU}(2)_{0}, \mathrm{SU}(2)_{1}, \mathrm{SU}(2)_{2}$, and $\mathrm{U}(1)_{Y}$ are denoted as $W_{0 \mu}^{a}, W_{1 \mu}^{a}, W_{2 \mu}^{a}$, and $B_{\mu}$, respectively $(a=1,2,3)$. The gauge couplings for each symmetry are denoted as $g_{0}, g_{1}, g_{2}$, and $g^{\prime}$, respectively. We summarize the matter fields and Higgs fields in table 1. Each fermion field corresponds to the $\mathrm{SM}$ fermion with the same $\mathrm{SU}(3)_{c}$ and $\mathrm{U}(1)_{Y}$ charge.

\footnotetext{
${ }^{1}$ The references for the other models are given in ref. [6].
} 


\begin{tabular}{|cc|ccccc|}
\hline field & spin & $\mathrm{SU}(3)_{c}$ & $\mathrm{SU}(2)_{0}$ & $\mathrm{SU}(2)_{1}$ & $\mathrm{SU}(2)_{2}$ & $\mathrm{U}(1)_{Y}$ \\
\hline$q_{L}$ & $\frac{1}{2}$ & 3 & 1 & 2 & 1 & $\frac{1}{6}$ \\
$u_{R}$ & $\frac{1}{2}$ & 3 & 1 & 1 & 1 & $\frac{2}{3}$ \\
$d_{R}$ & $\frac{1}{2}$ & 3 & 1 & 1 & 1 & $-\frac{1}{3}$ \\
$\ell_{L}$ & $\frac{1}{2}$ & 1 & 1 & 2 & 1 & $-\frac{1}{2}$ \\
$e_{R}$ & $\frac{1}{2}$ & 1 & 1 & 1 & 1 & -1 \\
\hline$H$ & 0 & 1 & 1 & 2 & 1 & $\frac{1}{2}$ \\
$\Phi_{1}$ & 0 & 1 & 2 & 2 & 1 & 0 \\
$\Phi_{2}$ & 0 & 1 & 1 & 2 & 2 & 0 \\
\hline
\end{tabular}

Table 1. The matter and Higgs fields and their gauge charges. The generation indices for the matter fields are implicit.

We introduce bi-fundamental scalar fields, $\Phi_{j}(j=1,2)$, expressed as the two-by-two matrices. We impose the real conditions for $\Phi_{j}$ to reduce the degrees of freedom.

$$
\Phi_{j}=-\epsilon \Phi_{j}^{*} \epsilon, \quad \text { where } \quad \epsilon=\left(\begin{array}{cc}
0 & 1 \\
-1 & 0
\end{array}\right)
$$

and each of $\Phi_{j}$ contains four real degrees of freedom. The gauge transformations of $\Phi_{j}$ and $H$ are shown below.

$$
\Phi_{1} \mapsto U_{0} \Phi_{1} U_{1}^{\dagger}, \quad \Phi_{2} \mapsto U_{2} \Phi_{2} U_{1}^{\dagger}, \quad H \mapsto e^{i \theta_{Y}} U_{1} H
$$

where $U_{n}$ denote the two-by-two gauge transformation matrices of $\mathrm{SU}(2)_{n}(n=0,1,2)$ and $\theta_{Y}$ is the phase of the $\mathrm{U}(1)_{Y}$. We also impose a discrete symmetry under the following transformation.

$$
\Phi_{1} \mapsto \Phi_{2}, \quad \Phi_{2} \mapsto \Phi_{1}, \quad W_{0 \mu}^{a} \mapsto W_{2 \mu}^{a}, \quad W_{2 \mu}^{a} \mapsto W_{0 \mu}^{a},
$$

where all the other fields remain unchanged. This transformation is equivalent to the exchange of $\mathrm{SU}(2)_{0}$ and $\mathrm{SU}(2)_{2}$, and thus it also requires $g_{0}=g_{2}$.

The Lagrangian for the extended bosonic sector is

$$
\begin{aligned}
\mathcal{L} \supset & -\frac{1}{4} B_{\mu \nu} B^{\mu \nu}-\sum_{j=0}^{2} \sum_{a=1}^{3} \frac{1}{4} W_{j \mu \nu}^{a} W_{j}^{a \mu \nu} \\
& +D_{\mu} H^{\dagger} D^{\mu} H+\frac{1}{2} \operatorname{tr} D_{\mu} \Phi_{1}^{\dagger} D_{\mu} \Phi_{1}+\frac{1}{2} \operatorname{tr} D_{\mu} \Phi_{2}^{\dagger} D_{\mu} \Phi_{2} \\
& -V_{\text {scalar }},
\end{aligned}
$$


where $V_{\text {scalar }}$ is the scalar potential as shown below.

$$
\begin{aligned}
V_{\text {scalar }}= & m^{2} H^{\dagger} H+m_{\Phi}^{2}\left[\operatorname{tr}\left(\Phi_{1}^{\dagger} \Phi_{1}\right)+\operatorname{tr}\left(\Phi_{2}^{\dagger} \Phi_{2}\right)\right] \\
& +\lambda\left(H^{\dagger} H\right)^{2}+\lambda_{\Phi}\left[\left(\operatorname{tr}\left(\Phi_{1}^{\dagger} \Phi_{1}\right)\right)^{2}+\left(\operatorname{tr}\left(\Phi_{2}^{\dagger} \Phi_{2}\right)\right)^{2}\right] \\
& +\lambda_{h \Phi} H^{\dagger} H\left[\operatorname{tr}\left(\Phi_{1}^{\dagger} \Phi_{1}\right)+\operatorname{tr}\left(\Phi_{2}^{\dagger} \Phi_{2}\right)\right]+\lambda_{12} \operatorname{tr}\left(\Phi_{1}^{\dagger} \Phi_{1}\right) \operatorname{tr}\left(\Phi_{2}^{\dagger} \Phi_{2}\right) .
\end{aligned}
$$

We assume the following vacuum expectation values (VEVs) to realize the $\mathrm{U}(1)_{\mathrm{em}}$ symmetry in the SM.

$$
\left\langle\Phi_{1}\right\rangle=\left\langle\Phi_{2}\right\rangle=\frac{1}{\sqrt{2}}\left(\begin{array}{cc}
v_{\Phi} & 0 \\
0 & v_{\Phi}
\end{array}\right), \quad\langle H\rangle=\left(\begin{array}{c}
0 \\
v \\
\sqrt{2}
\end{array}\right)
$$

where we take $v_{\Phi} \gg v$. We define component fields around the VEVs.

$$
\Phi_{j}=\left(\begin{array}{cc}
\frac{v_{\Phi}+\sigma_{j}+i \pi_{j}^{0}}{\sqrt{2}} & i \pi_{j}^{+} \\
i \pi_{j}^{-} & \frac{v_{\Phi}+\sigma_{j}-i \pi_{j}^{0}}{\sqrt{2}}
\end{array}\right), \quad H=\left(\begin{array}{c}
i \pi_{3}^{+} \\
\frac{v+\sigma_{3}-i \pi_{3}^{0}}{\sqrt{2}}
\end{array}\right) .
$$

After $\Phi_{1}$ and $\Phi_{2}$ develop the nonzero VEVs, we still have the residual $\mathrm{SU}(2) \times \mathrm{U}(1)_{Y}$ gauge symmetry whose gauge transformation is shown below.

$$
\Phi_{1} \mapsto U \Phi_{1} U^{\dagger}, \quad \quad \Phi_{2} \mapsto U \Phi_{2} U^{\dagger}, \quad H \mapsto e^{i \theta_{Y}} U H,
$$

where $U$ is the $\mathrm{SU}(2)$ two-by-two gauge transformation matrix. This $\mathrm{SU}(2)$ transformation corresponds to the $\mathrm{SU}(2)_{0} \times \mathrm{SU}(2)_{1} \times \mathrm{SU}(2)_{2}$ transformation with $U_{0}=U_{1}=U_{2} \equiv U$. This $\mathrm{SU}(2)$ is regarded as the $\mathrm{SU}(2)_{L}$ in the $\mathrm{SM}$ and simply called the $\mathrm{SU}(2)_{L}$ in the following discussion. The $\mathrm{SU}(2)_{L} \times \mathrm{U}(1)_{Y}$ symmetry is broken by $\langle H\rangle$ to the $\mathrm{U}(1)_{\text {em }}$ symmetry.

After all the Higgs fields develop nonzero VEVs, we still have the exchange symmetry whose symmetry transformations are shown below.

$$
\sigma_{1} \mapsto \sigma_{2}, \quad \sigma_{2} \mapsto \sigma_{1}, \quad W_{0 \mu}^{a} \mapsto W_{2 \mu}^{a}, \quad W_{2 \mu}^{a} \mapsto W_{0 \mu}^{a} .
$$

To find out a $Z_{2}$ parity from this residual discrete symmetry, we anti-symmetrize the exchanged fields and define the following states.

$$
\begin{aligned}
h_{D} & \equiv \frac{\sigma_{1}-\sigma_{2}}{\sqrt{2}}, \\
V_{\mu}^{0} & \equiv \frac{W_{0 \mu}^{0}-W_{2 \mu}^{0}}{\sqrt{2}}, \\
V_{\mu}^{ \pm} & \equiv \frac{W_{0 \mu}^{ \pm}-W_{2 \mu}^{ \pm}}{\sqrt{2}},
\end{aligned}
$$

where

$$
W_{n \mu}^{ \pm}=\frac{W_{n \mu}^{1} \mp i W_{n \mu}^{2}}{\sqrt{2}} \quad(n=0,2)
$$


These fields are eigenstates of the $\mathrm{U}(1)_{\mathrm{em}}$ charge. ${ }^{2}$ These states acquire $(-1)$ factors under the transformation in eq. (2.9) while all the other states remain unchanged. This is nothing but a $Z_{2}$ parity assignment. Note that the $Z_{2}$-odd states are automatically mass eigenstates since the mass mixing terms with other states are forbidden by this $Z_{2}$ parity.

We refer to $Z_{2}$-odd spin-1 particles, $V^{0}$ and $V^{ \pm}$, as " $V$-particles." The $V$-particles are approximately regarded as the spin-1 triplet of $\mathrm{SU}(2)_{L}$ and have the degenerated masses at the tree-level.

$$
m_{V^{0}}^{2}=m_{V_{ \pm}}^{2}=\frac{g_{0}^{2} v_{\Phi}^{2}}{4} \equiv m_{V}^{2}
$$

The electroweak radiative corrections break the degeneracy, which makes the charged component slightly heavier than the neutral one. We find the following value for mass splitting at the one-loop level.

$$
\delta m_{V} \equiv m_{V_{ \pm}}-m_{V_{0}} \simeq 168 \mathrm{MeV} .
$$

We assume $h_{D}$ is heavier than $V^{0}$ to focus on the spin-1 DM scenario. Therefore, $V^{0}$ is the lightest $Z_{2}$-odd particle and our stable spin-1 DM candidate.

\subsection{Physical spectrum}

We summarize the physical spectra in our model. Diagonalizing the mass matrices, we obtain the following mass eigenstates in the Higgs sector.

$$
\left(\begin{array}{c}
h_{D} \\
h \\
h^{\prime}
\end{array}\right)=\left(\begin{array}{ccc}
1 & 0 & 0 \\
0 & \sin \phi_{h} & \cos \phi_{h} \\
0 & \cos \phi_{h} & -\sin \phi_{h}
\end{array}\right)\left(\begin{array}{ccc}
\frac{1}{\sqrt{2}} & -\frac{1}{\sqrt{2}} & 0 \\
\frac{1}{\sqrt{2}} & \frac{1}{\sqrt{2}} & 0 \\
0 & 0 & 1
\end{array}\right)\left(\begin{array}{l}
\sigma_{1} \\
\sigma_{2} \\
\sigma_{3}
\end{array}\right) .
$$

We take the scalar masses, $\left\{m_{h}, m_{h^{\prime}}, m_{h_{D}}\right\}$, and the mixing angle, $\phi_{h}$, as input parameters. We can express the dimensionless couplings of $V_{\text {scalar }}$ in these input parameters as shown below.

$$
\begin{aligned}
\lambda & =\frac{m_{h}^{2} \cos ^{2} \phi_{h}+m_{h^{\prime}}^{2} \sin ^{2} \phi_{h}}{2 v^{2}}, \\
\lambda_{h \Phi} & =-\frac{\sin \phi_{h} \cos \phi_{h}}{2 \sqrt{2} v v_{\Phi}}\left(m_{h^{\prime}}^{2}-m_{h}^{2}\right), \\
\lambda_{\Phi} & =\frac{m_{h}^{2} \sin ^{2} \phi_{h}+m_{h^{\prime}}^{2} \cos ^{2} \phi_{h}+m_{h_{D}}^{2}}{16 v_{\Phi}^{2}}, \\
\lambda_{12} & =\frac{m_{h}^{2} \sin ^{2} \phi_{h}+m_{h^{\prime}}^{2} \cos ^{2} \phi_{h}-m_{h_{D}}^{2}}{8 v_{\Phi}^{2}} .
\end{aligned}
$$

In the following discussion, we focus on $\phi_{h} \lesssim 0.1$ to evade the direct detection constraints [15].

\footnotetext{
${ }^{2}$ The $\mathrm{U}(1)_{\mathrm{em}}$ symmetry generator is expressed as $Q=T_{0}^{3}+T_{1}^{3}+T_{2}^{3}+Y$ where $T_{n}^{3}$ denote the third generators of $\mathrm{SU}(2)_{n}(n=0,1,2)$.
} 
For the charged gauge bosons, we obtain

$$
\left(\begin{array}{c}
V_{\mu}^{ \pm} \\
W_{\mu}^{ \pm} \\
W_{\mu}^{\prime \pm}
\end{array}\right)=\left(\begin{array}{ccc}
1 & 0 & 0 \\
0 & \cos \phi_{ \pm} & \sin \phi_{ \pm} \\
0 & -\sin \phi_{ \pm} & \cos \phi_{ \pm}
\end{array}\right)\left(\begin{array}{ccc}
\frac{1}{\sqrt{2}} & 0 & -\frac{1}{\sqrt{2}} \\
0 & 1 & 0 \\
\frac{1}{\sqrt{2}} & 0 & \frac{1}{\sqrt{2}}
\end{array}\right)\left(\begin{array}{c}
W_{0 \mu}^{ \pm} \\
W_{1 \mu}^{ \pm} \\
W_{2 \mu}^{\mu}
\end{array}\right)
$$

where

$$
\cos \phi_{ \pm}=\sqrt{\frac{m_{V^{ \pm}}^{2}-m_{W}^{2}}{m_{W^{\prime}}^{2}-m_{W}^{2}}}, \quad \sin \phi_{ \pm}=\sqrt{\frac{m_{W^{\prime}}^{2}-m_{V^{ \pm}}^{2}}{m_{W^{\prime}}^{2}-m_{W}^{2}}} .
$$

The masses of charged $Z_{2}$-even vectors are obtained as

$$
\begin{aligned}
& m_{W}^{2}=\frac{1}{8}\left\{g_{1}^{2} v^{2}+\left(g_{0}^{2}+2 g_{1}^{2}\right) v_{\Phi}^{2}-\sqrt{-4 g_{0}^{2} g_{1}^{2} v^{2} v_{\Phi}^{2}+\left[g_{1}^{2} v^{2}+\left(g_{0}^{2}+2 g_{1}^{2}\right) v_{\Phi}^{2}\right]^{2}}\right\} \\
& m_{W^{\prime}}^{2}=\frac{1}{8}\left\{g_{1}^{2} v^{2}+\left(g_{0}^{2}+2 g_{1}^{2}\right) v_{\Phi}^{2}+\sqrt{-4 g_{0}^{2} g_{1}^{2} v^{2} v_{\Phi}^{2}+\left[g_{1}^{2} v^{2}+\left(g_{0}^{2}+2 g_{1}^{2}\right) v_{\Phi}^{2}\right]^{2}}\right\}
\end{aligned}
$$

For the neutral gauge bosons, we obtain

$$
\left(\begin{array}{c}
V_{\mu}^{0} \\
A_{\mu} \\
Z_{\mu} \\
Z_{\mu}^{\prime}
\end{array}\right)=\left(\begin{array}{cccc}
\frac{1}{\sqrt{2}} & 0 & -\frac{1}{\sqrt{2}} & 0 \\
\frac{e}{g_{0}} & \frac{e}{g_{1}} & \frac{e}{g_{0}} & \frac{e}{g^{\prime}} \\
\omega_{Z}^{0} & \omega_{Z}^{1} & \omega_{Z}^{0} & \omega_{Z}^{B} \\
\omega_{Z^{\prime}}^{0} & \omega_{Z^{\prime}}^{1} & \omega_{Z^{\prime}}^{0} & \omega_{Z^{\prime}}^{B}
\end{array}\right)\left(\begin{array}{c}
W_{0 \mu}^{3} \\
W_{1 \mu}^{3} \\
W_{2 \mu}^{3} \\
B_{\mu}
\end{array}\right)
$$

where

$$
\begin{aligned}
e & =\left(\frac{2}{g_{0}^{2}}+\frac{1}{g_{1}^{2}}+\frac{1}{g^{\prime 2}}\right)^{-1 / 2}, \\
\omega_{Z}^{0} & =\frac{e g_{1}}{\sqrt{g_{0}^{2}+2 g_{1}^{2}} g^{\prime}} \cos \phi_{0}+\frac{g_{0}}{\sqrt{2\left(g_{0}^{2}+2 g_{1}^{2}\right)}} \sin \phi_{0}, \\
\omega_{Z}^{1} & =\frac{e g_{0}}{\sqrt{g_{0}^{2}+2 g_{1}^{2}} g^{\prime}} \cos \phi_{0}-\frac{\sqrt{2} g_{1}}{\sqrt{g_{0}^{2}+2 g_{1}^{2}}} \sin \phi_{0}, \\
\omega_{Z}^{B} & =-\frac{e \sqrt{g_{0}^{2}+2 g_{1}^{2}}}{g_{0} g_{1}} \cos \phi_{0}, \\
\omega_{Z^{\prime}}^{0} & =\frac{g_{0}}{\sqrt{2\left(g_{0}^{2}+2 g_{1}^{2}\right)}} \cos \phi_{0}-\frac{e g_{1}}{\sqrt{g_{0}^{2}+2 g_{1}^{2}} g^{\prime}} \sin \phi_{0}, \\
\omega_{Z^{\prime}}^{1} & =-\frac{\sqrt{2} g_{1}}{\sqrt{g_{0}^{2}+2 g_{1}^{2}}} \cos \phi_{0}-\frac{e g_{0}}{\sqrt{g_{0}^{2}+2 g_{1}^{2}} g^{\prime}} \sin \phi_{0}, \\
\omega_{Z^{\prime}}^{B} & =\frac{e \sqrt{g_{0}^{2}+2 g_{1}^{2}}}{g_{0} g_{1}} \sin \phi_{0} .
\end{aligned}
$$


We define $\phi_{0}$ that satisfies the following relation.

$$
\frac{1}{4}\left(\begin{array}{cc}
\frac{g_{0}^{2} g_{1}^{2} g^{\prime 2}}{e^{2}\left(g_{0}^{2}+2 g_{1}^{2}\right)} v^{2} & -\frac{\sqrt{2} g_{0} g_{1}^{3} g^{\prime}}{e\left(g_{0}^{2}+2 g_{1}^{2}\right)} v^{2} \\
-\frac{\sqrt{2} g_{0} g_{1}^{3} g^{\prime}}{e\left(g_{0}^{2}+2 g_{1}^{2}\right)} v^{2} & \frac{\left(g_{0}^{2}+2 g_{1}^{2}\right)^{2} v_{\Phi}^{2}+2 g_{1}^{4} v^{2}}{\left(g_{0}^{2}+2 g_{1}^{2}\right)}
\end{array}\right)\left(\begin{array}{cc}
\cos \phi_{0} & -\sin \phi_{0} \\
\sin \phi_{0} & \cos \phi_{0}
\end{array}\right)=\left(\begin{array}{cc}
\cos \phi_{0} & -\sin \phi_{0} \\
\sin \phi_{0} & \cos \phi_{0}
\end{array}\right)\left(\begin{array}{cc}
m_{Z}^{2} & 0 \\
0 & m_{Z^{\prime}}^{2}
\end{array}\right) .
$$

The parameters in the Lagrangian in eq. (2.4) are summarized below.

$$
\left\{g_{0}, g_{1}, g^{\prime}, m^{2}, m_{\Phi}^{2}, \lambda, \lambda_{\Phi}, \lambda_{h \Phi}, \lambda_{12}\right\} .
$$

We choose the following input parameters for our phenomenological study.

$$
\left\{e, m_{Z}, v, m_{h}, m_{Z^{\prime}}, m_{V}, m_{h^{\prime}}, m_{h_{D}}, \phi_{h}\right\} .
$$

The first four parameters are already fixed by the experiments, while the last five parameters are free. As we mentioned before, we consider the small $\phi_{h}$ regime. Therefore, the SM Higgs coupling with the $V$-particles are suppressed by the small mixing factor, $\sin \phi_{h}$. Although we do not specify the values of $m_{h^{\prime}}$ and $m_{h_{D}}$ in the following discussion, we assume these scalar masses are in the $\mathrm{TeV}$ scale to focus on the phenomenological aspects resulting from the electroweak interactions. The constraints on these parameters in the scalar sector are studied in ref. [15]. For later convenience, we introduce $g_{W}$ as defined below.

$$
g_{W} \equiv\left(\frac{2}{g_{0}^{2}}+\frac{1}{g_{1}^{2}}\right)^{-1 / 2}
$$

This coupling corresponds to the $\mathrm{SU}(2)_{L}$ gauge coupling approximately, and we obtain $g_{W} \simeq 0.65$, which is the same value as the $\mathrm{SU}(2)_{L}$ gauge coupling in the $\mathrm{SM}$, in the limit of $v_{\Phi} \gg v$.

We assume $v_{\Phi} \gg v$ throughout our study, and it is useful to derive the approximated forms for the physical values in this limit. For $m_{W}$ and $m_{W^{\prime}}$, we obtain

$$
\begin{aligned}
& m_{W} \simeq \frac{g_{W} v}{2}, \\
& m_{W^{\prime}} \simeq m_{Z^{\prime}},
\end{aligned}
$$

and thus we easily obtain the correct value of $m_{W}$. The gauge couplings, $g_{0}$ and $g_{1}$, are expressed as

$$
\begin{aligned}
& g_{0} \simeq \sqrt{2} g_{W} \frac{m_{Z^{\prime}}}{m_{V}} \frac{1}{\sqrt{\frac{m_{Z^{\prime}}^{2}}{m_{V}^{2}}-1}}, \\
& g_{1} \simeq g_{W} \frac{m_{Z^{\prime}}}{m_{V}} .
\end{aligned}
$$

These couplings are constrained by the perturbative unitarity bounds. We obtain the following constraints in the high energy regime [24].

$$
g_{j}<\sqrt{\frac{16 \pi}{\sqrt{6}}} \simeq 4.53 \quad(j=0,1) .
$$


From these bounds, we can narrow down the viable range of the mass ratio, $\frac{m_{Z^{\prime}}}{m_{V}}$. If we take $g_{W} \simeq 0.65$, we obtain

$$
1.02 \lesssim \frac{m_{Z^{\prime}}}{m_{V}} \lesssim 6.97
$$

More detailed explanations of our model are given in ref. [15].

\subsection{Couplings of $V$-particles}

We show the couplings of the $V$-particles in the limit of $v_{\Phi} \gg v$. The vector triple couplings are shown below.

$$
\mathcal{L} \supset i C_{X Y Z}\left[\left(X_{\nu}^{+} \overleftrightarrow{\partial}^{\mu} Y^{-\nu}\right) Z_{\mu}^{0}+\left(Y_{\nu}^{-} \overleftrightarrow{\partial}^{\mu} Z^{0 \nu}\right) X_{\mu}^{+}+\left(Z_{\nu}^{0} \overleftrightarrow{\partial}^{\mu} X^{+\nu}\right) Y_{\mu}^{-}\right]
$$

where

$$
\begin{aligned}
& C_{W^{+} V^{-} V^{0}}=C_{V^{+} W^{-} V^{0}} \simeq g_{W}, \\
& C_{V^{+} V^{-}}=e, \\
& C_{V+V-Z} \simeq g_{W} c_{W}, \\
& C_{V^{+} V^{-} Z^{\prime}} \simeq \frac{g_{W}}{\sqrt{\frac{m_{Z^{\prime}}^{2}}{m_{V}^{2}}-1}} \equiv g_{Z^{\prime}} .
\end{aligned}
$$

We define $s_{W} \equiv \sin \theta_{W}$ and $c_{W} \equiv \cos \theta_{W}$ where $\theta_{W}$ is the Weinberg angle. We also define $g_{Z^{\prime}}$ in eq. (2.47) to characterize the couplings between $Z^{\prime}$ and the $V$-particles. The vector quartic couplings are shown below.

$$
\mathcal{L} \supset C_{X Y Z W} X_{\rho}^{+} Y_{\sigma}^{-} Z_{\mu}^{0} W_{\nu}^{0}\left(g^{\rho \mu} g^{\sigma \nu}+g^{\rho \nu} g^{\sigma \mu}-2 g^{\rho \sigma} g^{\mu \nu}\right),
$$

where

$$
\begin{aligned}
C_{V^{+} V^{-}} A A & =\frac{e^{2}}{2} \\
C_{V^{+} V^{-}} A Z & \simeq e g_{W} c_{W}, \\
C_{V^{+} V^{-}} A Z^{\prime} & =e C_{V^{+} V^{-} Z^{\prime}}
\end{aligned}
$$

\section{Annihilation cross section for $V$-particles}

As studied in the previous work [15], we need $m_{V} \gtrsim \mathcal{O}(1) \mathrm{TeV}$ to explain the correct DM relic abundance in our model. In this region, the $W$ and $Z$ bosons effectively form the longrange force potential for the $V$-particles. Consequently, we have the sizable Sommerfeld enhancement in the DM annihilation processes. For this class of models, the monochromatic gamma-ray line from the DM annihilation is an excellent probe.

In this section, we summarize the formulas to discuss the gamma-ray line signature from our spin-1 DM. We apply the same formalism as those for the spin- 0 or spin- $1 / 2$ DM with electroweak interactions [4, 20]. First, we define the non-relativistic (NR) field operators for the $V$-particles to fix our notations. Second, we show the NR two-body effective action for the $V$-particles. We focus on the electrically neutral two-body states to study the DM annihilation signals. We derive the effective action in appendix A. 


\subsection{Non-relativistic field operators for $V$-particles}

The asymptotic field operators for the $V$-particles are defined below.

$$
\begin{aligned}
V_{\mu}^{0}(x) & =\sum_{A=1}^{3} \int \frac{d^{3} p}{(2 \pi)^{3}} \frac{1}{\sqrt{2 E_{\mathrm{p}}}}\left[a^{A}(\boldsymbol{p}) e^{-i p \cdot x} \epsilon_{\mu}^{A}(p)+a^{A \dagger}(\boldsymbol{p}) e^{i p \cdot x} \epsilon_{\mu}^{A *}(p)\right], \\
V_{\mu}^{-}(x) & =\sum_{A=1}^{3} \int \frac{d^{3} p}{(2 \pi)^{3}} \frac{1}{\sqrt{2 E_{\mathrm{p}}}}\left[b^{A}(\boldsymbol{p}) e^{-i p \cdot x} \epsilon_{\mu}^{A}(p)+d^{A \dagger}(\boldsymbol{p}) e^{i p \cdot x} \epsilon_{\mu}^{A *}(p)\right], \\
V_{\mu}^{+}(x) & =\sum_{A=1}^{3} \int \frac{d^{3} p}{(2 \pi)^{3}} \frac{1}{\sqrt{2 E_{\mathrm{p}}}}\left[d^{A}(\boldsymbol{p}) e^{-i p \cdot x} \epsilon_{\mu}^{A}(p)+b^{A \dagger}(\boldsymbol{p}) e^{i p \cdot x} \epsilon_{\mu}^{A *}(p)\right],
\end{aligned}
$$

where $\epsilon_{\mu}^{A}(p)$ denote the physical polarization vectors. These polarization vectors satisfy the transverse conditions,

$$
p^{\mu} \epsilon_{\mu}^{A}(p)=0
$$

and the orthogonal relations.

$$
\epsilon_{\mu}^{A *}(p) \epsilon^{B \mu}(p)=-\delta^{A B} .
$$

We impose the canonical commutation relations between the annihilation and creation operators.

$$
\begin{aligned}
{\left[a^{A}(\boldsymbol{p}), a^{B \dagger}\left(\boldsymbol{p}^{\prime}\right)\right] } & =(2 \pi)^{3} \delta^{3}\left(\boldsymbol{p}-\boldsymbol{p}^{\prime}\right) \delta^{A B}, \\
{\left[b^{A}(\boldsymbol{p}), b^{B \dagger}\left(\boldsymbol{p}^{\prime}\right)\right] } & =(2 \pi)^{3} \delta^{3}\left(\boldsymbol{p}-\boldsymbol{p}^{\prime}\right) \delta^{A B}, \\
{\left[d^{A}(\boldsymbol{p}), d^{B \dagger}\left(\boldsymbol{p}^{\prime}\right)\right] } & =(2 \pi)^{3} \delta^{3}\left(\boldsymbol{p}-\boldsymbol{p}^{\prime}\right) \delta^{A B} .
\end{aligned}
$$

To derive the NR two-body effective action, we perform the NR expansion for these operators and integrate out the large momentum modes. Since the zeroth component of the polarization tensor is sub-leading, $\mathcal{O}\left(|\boldsymbol{p}| / m_{V}\right)$, we focus on the spacial components $(i=1,2,3)$ for the $V$-particle operators.

$$
\begin{aligned}
V_{i}^{0}(x) & \simeq \frac{1}{\sqrt{2 m_{V}}}\left[e^{-i m_{V} t} \mathcal{A}_{i}(x)+e^{i m_{V} t} \mathcal{A}_{i}^{\dagger}(x)\right], \\
V_{i}^{-}(x) & \simeq \frac{1}{\sqrt{2 m_{V}}}\left[e^{-i m_{V} t} \mathcal{B}_{i}(x)+e^{i m_{V} t} \mathcal{D}_{i}^{\dagger}(x)\right], \\
V_{i}^{+}(x) & \simeq \frac{1}{\sqrt{2 m_{V}}}\left[e^{-i m_{V} t} \mathcal{D}_{i}(x)+e^{i m_{V} t} \mathcal{B}_{i}^{\dagger}(x)\right],
\end{aligned}
$$

where we define

$$
\begin{aligned}
& \mathcal{A}_{i}(x)=\sum_{A=1}^{3} \int \frac{d^{3} p}{(2 \pi)^{3}} a^{A}(\boldsymbol{p}) e^{-i p \cdot x} \epsilon_{i}^{A}(p), \\
& \mathcal{B}_{i}(x)=\sum_{A=1}^{3} \int \frac{d^{3} p}{(2 \pi)^{3}} b^{A}(\boldsymbol{p}) e^{-i p \cdot x} \epsilon_{i}^{A}(p), \\
& \mathcal{D}_{i}(x)=\sum_{A=1}^{3} \int \frac{d^{3} p}{(2 \pi)^{3}} d^{A}(\boldsymbol{p}) e^{-i p \cdot x} \epsilon_{i}^{A}(p) .
\end{aligned}
$$


We also define the NR one particle states for the $V$-particles with the momentum $\boldsymbol{p}$ and the polarization $A$.

$$
\begin{aligned}
\left|V^{0} ; \boldsymbol{p}, A\right\rangle & \simeq \sqrt{2 m_{V}} a^{A \dagger}(\boldsymbol{p})|0\rangle, \\
\left|V^{-} ; \boldsymbol{p}, A\right\rangle & \simeq \sqrt{2 m_{V}} b^{A \dagger}(\boldsymbol{p})|0\rangle, \\
\left|V^{+} ; \boldsymbol{p}, A\right\rangle & \simeq \sqrt{2 m_{V}} d^{A \dagger}(\boldsymbol{p})|0\rangle .
\end{aligned}
$$

\subsection{Two-body effective action}

We show the two-body effective action for the NR $V$-particles. The electrically neutral two-body states are composed of $V^{-}(t, \boldsymbol{x}) V^{+}(t, \boldsymbol{y})$ or $V^{0}(t, \boldsymbol{x}) V^{0}(t, \boldsymbol{y})$. We change the space-time coordinates into the center of mass coordinate, $R=\left(R^{0}, \boldsymbol{R}\right)$, and the relative coordinate, $r$.

$$
R^{0}=t, \quad \boldsymbol{R} \equiv \frac{\boldsymbol{x}+\boldsymbol{y}}{2}, \quad \boldsymbol{r} \equiv \boldsymbol{x}-\boldsymbol{y} .
$$

As shown later, the NR leading-order potential has a spherically symmetric form. Therefore, the total spin angular momentum, $J$, and $z$-component of the spin angular momentum, $J_{z}$, are good quantum numbers. We obtain the following effective action in the decomposed form into each partial wave state.

$$
S_{\mathrm{eff}}=\sum_{J, J_{z}} \int d^{4} R d^{3} r \Phi^{J, J_{z} \dagger}(R, \boldsymbol{r}) \cdot\left[\left(i \partial_{R^{0}}+\frac{\nabla_{R}^{2}}{4 m_{V}}+\frac{\nabla_{r}^{2}}{m_{V}}\right)-\hat{V}(r)+i \frac{9}{2} \hat{\Gamma}^{J} \delta^{3}(\boldsymbol{r})\right] \cdot \Phi^{J, J_{z}}(R, \boldsymbol{r}),
$$

where $r \equiv|\boldsymbol{r}|$ and $J=0,1,2$ with $\left|J_{z}\right| \leq J$. The $\hat{V}$ and $\hat{\Gamma}^{J}$ denote the real part and imaginary part of the potential, respectively. We define the two-body fields for each $\left(J, J_{z}\right)$ as shown below.

$$
\Phi^{J, J_{z}}(R, \boldsymbol{r})=\left(\begin{array}{c}
\phi_{C}^{J, J_{z}}(R, \boldsymbol{r}) \\
\phi_{N}^{J, J_{z}}(R, \boldsymbol{r})
\end{array}\right)
$$

The upper and lower components are formed by $V^{-} V^{+}$and $V^{0} V^{0}$, respectively.

$$
\begin{array}{ll}
\phi_{C}^{J, J_{z}}(R, \boldsymbol{r})=\mathcal{B}_{i}\left(R^{0}, \boldsymbol{R}+\boldsymbol{r} / 2\right) S_{i j}^{J, J_{z}} \mathcal{D}_{j}\left(R^{0}, \boldsymbol{R}-\boldsymbol{r} / 2\right) & (J=0,1,2), \\
\phi_{N}^{J, J_{z}}(R, \boldsymbol{r})=\frac{1}{\sqrt{2}} \mathcal{A}_{i}\left(R^{0}, \boldsymbol{R}+\boldsymbol{r} / 2\right) S_{i j}^{J, J_{z}} \mathcal{A}_{j}\left(R^{0}, \boldsymbol{R}-\boldsymbol{r} / 2\right) & (J=0,2) .
\end{array}
$$

In the above definition, we introduce the basis of three-by-three matrices.

$$
\hat{S}^{J, J_{z}} \equiv S_{i j}^{J, J_{z}} \quad(i, j=1,2,3),
$$


where

$$
\begin{aligned}
& \hat{S}^{0,0}=\frac{-1}{\sqrt{3}}\left(\begin{array}{lll}
1 & 0 & 0 \\
0 & 1 & 0 \\
0 & 0 & 1
\end{array}\right) \\
& \hat{S}^{1,1}=\frac{1}{2}\left(\begin{array}{ccc}
0 & 0 & -1 \\
0 & 0 & -i \\
1 & i & 0
\end{array}\right), \quad \hat{S}^{1,0}=\frac{1}{\sqrt{2}}\left(\begin{array}{ccc}
0 & i & 0 \\
-i & 0 & 0 \\
0 & 0 & 0
\end{array}\right), \quad \hat{S}^{1,-1}=\frac{1}{2}\left(\begin{array}{ccc}
0 & 0 & -1 \\
0 & 0 & i \\
1 & -i & 0
\end{array}\right), \\
& \hat{S}^{2,2}=\frac{1}{2}\left(\begin{array}{ccc}
1 & i & 0 \\
i & -1 & 0 \\
0 & 0 & 0
\end{array}\right), \quad \hat{S}^{2,1}=\frac{1}{2}\left(\begin{array}{ccc}
0 & 0 & -1 \\
0 & 0 & -i \\
-1 & -i & 0
\end{array}\right), \quad \hat{S}^{2,0}=\frac{1}{\sqrt{6}}\left(\begin{array}{ccc}
-1 & 0 & 0 \\
0 & -1 & 0 \\
0 & 0 & 2
\end{array}\right) \text {, } \\
& \hat{S}^{2,-1}=\frac{1}{2}\left(\begin{array}{ccc}
0 & 0 & 1 \\
0 & 0 & -i \\
1 & -i & 0
\end{array}\right), \quad \hat{S}^{2,-2}=\frac{1}{2}\left(\begin{array}{ccc}
1 & -i & 0 \\
-i & -1 & 0 \\
0 & 0 & 0
\end{array}\right) \text {. }
\end{aligned}
$$

These matrices satisfy the following orthogonal relation.

$$
\operatorname{tr}\left[\hat{S}^{J, J_{z}} \hat{S}^{J^{\prime}, J_{z}^{\prime}}\right]=(-1)^{J} \delta^{J J^{\prime}} \delta^{J_{z} J_{z}^{\prime}}
$$

Note that $\hat{S}^{J, J_{z}}$ is symmetric matrices for $J=0,2$ and anti-symmetric for $J=1$. We have no $\phi_{N}^{1, J_{z}}$ because $\phi_{N}$ is composed of identical particles. As mentioned before, $J$ is conserved as long as we focus on the NR leading order contributions. Therefore, $J=1$ partial wave modes are irrelevant to discuss the DM annihilation signals. We only consider $J=0,2$ states in the following discussion. The normalization factors for the two-body states are fixed to realize the canonical weights for the two-body propagators. The twobody propagator of $\phi_{C}^{J, J_{z}}$ is defined as the time-ordered product.

$$
\left\langle 0\left|T \phi_{C}^{J, J_{z}}(R, \boldsymbol{r}) \phi_{C}^{J, J_{z} \dagger}(0, \mathbf{0})\right| 0\right\rangle=\int \frac{d^{3} P}{(2 \pi)^{3}} \frac{d^{3} k}{(2 \pi)^{3}} e^{-i\left(\frac{|\boldsymbol{P}|^{2}}{4 m}+\frac{\boldsymbol{k}^{2}}{m}\right) R^{0}+i \boldsymbol{P} \cdot \boldsymbol{R}} e^{+i \boldsymbol{k} \cdot \boldsymbol{r}} \theta\left(R^{0}\right)
$$

where $\theta\left(R^{0}\right)$ is the Heaviside step function. This expression is obtained by substituting the explicit forms of $\phi_{C}^{J, J_{z}}$ and $\phi_{C}^{J, J_{z} \dagger}$ and using the canonical commutation relation between them. ${ }^{3}$ The definition of $\phi_{N}^{J, J_{z}}$ has another normalization factor of $\frac{1}{\sqrt{2}}$ because $\phi_{N}^{J, J_{z}}$ is composed of identical particles.

\footnotetext{
${ }^{3}$ Note that $\left(\hat{S}^{J, J_{z}}\right)^{\dagger}=(-1)^{J}\left(\hat{S}^{J, J_{z}}\right)^{*}$ because it is symmetric for $J=0,2$ but anti-symmetric for $J=1$.
} 
The leading-order expression for $\hat{V}$ is obtained as follows.

$$
\hat{V}(r)=\left(\begin{array}{cc}
2 \delta m_{V}-\frac{\alpha_{2} s_{W}^{2}}{r}-\frac{\alpha_{2} c_{W}^{2} e^{-m_{Z} r}}{r} & -\frac{\sqrt{2} \alpha_{2} e^{-m_{W} r}}{r} \\
-\frac{\sqrt{2} \alpha_{2} e^{-m_{W} r}}{r} & 0
\end{array}\right),
$$

where $\alpha_{2} \equiv \frac{g_{W}^{2}}{4 \pi}$. This potential is induced by the NR processes between $V$-particles. The off-diagonal elements are induced by the $W$ boson exchange processes, which cause the mixing between $\phi_{C}$ and $\phi_{N}$. Compared with the leading-order contributions, the Higgs exchange contributions are suppressed by small $\phi_{h}$. The $W^{\prime}$ and $Z^{\prime}$ exchange contributions are exponentially suppressed by $m_{W^{\prime}}$ and $m_{Z^{\prime}}$. The contributions from the vector quadratic couplings are suppressed by $\frac{1}{m_{V}^{2}}$. The leading-order expression for $\hat{V}$ is the same as that of the $\mathrm{SU}(2)_{L}$ triplet DM with spin-0/spin- $\frac{1}{2}$. This is because the spin-dependent features decouple from the NR processes. We show the derivation of $\hat{V}$ in appendix A.1.

The imaginary part of the potential, $\hat{\Gamma}^{J}$, is derived by operator matching between the two-body field operators and the calculations of the loop amplitudes of the $V$-particles. ${ }^{4}$ We focus on the one-loop diagrams with the intermediate states, $X X^{\prime}=\gamma \gamma, Z \gamma, Z^{\prime} \gamma$, involving the gamma-ray line signatures. The annihilation modes into $h \gamma$ and $h^{\prime} \gamma$ only induce $J=1$ partial wave contributions, which is irrelevant to the DM annihilation signals. We decompose $\hat{\Gamma}^{J}$ into each contribution of the intermediate state, $X X^{\prime}$, as denoted by $\Gamma_{X X^{\prime}}^{J}$. The leading-order expressions are shown below.

$$
\begin{aligned}
& \hat{\Gamma}_{\gamma \gamma}^{J=0}=\frac{2}{3} \frac{\pi \alpha_{2}^{2}}{m_{V}^{2}}\left(\begin{array}{cc}
s_{W}^{4} & 0 \\
0 & 0
\end{array}\right), \\
& \hat{\Gamma}_{\gamma \gamma}^{J=2}=\frac{32}{45} \frac{\pi \alpha_{2}^{2}}{m_{V}^{2}}\left(\begin{array}{cc}
s_{W}^{4} & 0 \\
0 & 0
\end{array}\right) \text {, } \\
& \hat{\Gamma}_{Z \gamma}^{J=0}=\frac{2}{3} \frac{\pi \alpha_{2}^{2}}{m_{V}^{2}}\left(\begin{array}{cc}
2 c_{W}^{2} s_{W}^{2} & 0 \\
0 & 0
\end{array}\right) \text {, } \\
& \hat{\Gamma}_{Z \gamma}^{J=2}=\frac{32}{45} \frac{\pi \alpha_{2}^{2}}{m_{V}^{2}}\left(\begin{array}{cc}
2 c_{W}^{2} s_{W}^{2} & 0 \\
0 & 0
\end{array}\right) \text {, } \\
& \hat{\Gamma}_{Z^{\prime} \gamma}^{J=0}=\frac{1}{27} \frac{\alpha_{2} g_{Z^{\prime}}^{2}}{m_{V}^{2}}\left(1-r_{Z^{\prime}}\right)\left(3-2 r_{Z^{\prime}}\right)^{2}\left(\begin{array}{cc}
s_{W}^{2} & 0 \\
0 & 0
\end{array}\right), \\
& \hat{\Gamma}_{Z^{\prime} \gamma}^{J=2}=\frac{8}{135} \frac{\alpha_{2} g_{Z^{\prime}}^{2}}{m_{V}^{2}}\left(1-r_{Z^{\prime}}\right)\left(6+3 r_{Z^{\prime}}+r_{Z^{\prime}}^{2}\right)\left(\begin{array}{cc}
s_{W}^{2} & 0 \\
0 & 0
\end{array}\right) \text {, }
\end{aligned}
$$

\footnotetext{
${ }^{4}$ We choose the factor of " $\frac{9}{2}$ " in eq. (3.19) so that the $(1,1)$-component of $\hat{\Gamma}^{J}$ is equal to the tree-level spin-averaged velocity-weighted annihilation cross sections of $V^{-} V^{+}$with $J$.
} 
where $r_{Z^{\prime}} \equiv \frac{m_{Z^{\prime}}^{2}}{4 m_{V}^{2}}$, and $g_{Z^{\prime}}$ is defined in eq. (2.47). We show the derivation of $\hat{\Gamma}^{J}$ in appendix A.2.

\subsection{Annihilation cross section}

We derive the $s$-wave spin-averaged velocity-weighted cross section for $V^{0} V^{0} \rightarrow X X^{\prime}$ annihilation process through the optical theorem.

$$
\left\langle\sigma v_{\mathrm{rel}}\right\rangle_{X X^{\prime}}=C \sum_{\alpha, \beta} \sum_{J, J_{z}}\left(\Gamma_{X X^{\prime}}^{J}\right)_{\alpha \beta} d_{2 \alpha}(E) d_{2 \beta}^{*}(E),
$$

where $C=2$ for the initial states composed of the identical particles. We introduce $E \simeq \frac{m_{V} v_{\text {rel }}^{2}}{4}$ as the NR kinetic energy of the $V$-particles, and $d_{\alpha \beta}(E)(\alpha, \beta=1,2)$ as the Sommerfeld enhancement factor. We numerically obtain $d_{\alpha \beta}(E)$ by solving the Schrödinger equation [20].

Before we show the numerical results, we remark the distinctive features of our spin-1 DM compared with the Wino DM, which is $\mathrm{SU}(2)_{L}$ triplet spin- $\frac{1}{2} \mathrm{DM}$. We focus on the leading-order results in both DM systems to show the comparison. ${ }^{5}$ The Sommerfeld enhancement factor is approximately the same in both systems since we have the same $\hat{V}$ and the mass splitting at the leading-order. This is because the leading-order interactions with $W$ and $Z$ bosons in the NR limit are independent of the DM spin. We have contributions other than $W$ and $Z$ interactions but they are sub-leading as mentioned above. The SM Higgs contributions for $\hat{V}$, which is zero for pure Wino DM at the leading-order, are suppressed by the small mixing angle $\phi_{h}$. The contributions from $W^{\prime}, Z^{\prime}$, and $h^{\prime}$ are suppressed by their masses. ${ }^{6}$ Consequently, we have the same resonance structure as that in the Wino system. On the other hand, the annihilation cross section into $\gamma \gamma$ and $Z \gamma$ are larger than those for the Wino by $\frac{38}{9} \simeq 4.22 \cdots$. This is because spin- 1 DM forms both $J=0$ and $J=2$ partial wave states while spin- $\frac{1}{2}$ DM forms only $J=0$ state. Our spin-1 DM also has a new annihilation mode into $Z^{\prime} \gamma$. If this new channel is kinematically opened, a photon is emitted with the energy depending on both $m_{V}$ and $m_{Z^{\prime}}$. This channel also contributes to the monochromatic gamma-ray line signals, which we study further in section 4.

\section{Gamma-ray line signatures}

We study the gamma-ray line signatures from our spin-1 DM. We derive the constraints from the search for monochromatic gamma-ray lines in the Galactic Center region. The derived constraints highly depend on the DM density profiles, and thus we show our result by taking some types of benchmark profiles to show the uncertainty.

\footnotetext{
${ }^{5}$ For the Wino DM, the Sudakov log corrections [25-29] are precisely evaluated. In our spin-1 DM system, those potentially large corrections have not been evaluated yet, and thus our predictions have the uncertainty of $\mathcal{O}(1)$ factors. The one-loop correction for the real part of the potential in the Wino DM system is studied in refs. [30, 31].

${ }^{6}$ Even if we consider a relatively light $h^{\prime}$ and take $m_{h^{\prime}} \sim 1 \mathrm{TeV}$, the contribution to the potential is suppressed by $\exp \left(-m_{h^{\prime}} / m_{Z}\right) \sim \exp (-10)$. If we take a much lighter value of $m_{h^{\prime}}$, we have to study the constraint on the $h^{\prime}$ from the low energy experiments, which is beyond the scope of this paper.
} 


\subsection{Line cross section}

The latest analysis for the gamma-ray line signals is performed by the H.E.S.S. collaboration using the ten years data of the gamma-ray observation in the Galactic Center region [32]. We constrain the gamma-ray line signatures from the DM annihilation by using this result. In our model, we have three annihilation modes involving the gamma-ray line signals, $\left\{\gamma \gamma, Z \gamma, Z^{\prime} \gamma\right\}$. For the $\gamma \gamma / Z \gamma$ modes, the photon energy is approximately equal to the DM mass, $E_{\gamma} \simeq m_{V}$, where we take the NR limit for the initial DM pair and neglect $m_{Z}$. For the $Z^{\prime} \gamma$ mode, we can not neglect $m_{Z^{\prime}}$ because $m_{Z^{\prime}}$ must be heavier than $m_{V}$, see eq. (2.42). The photon energy in the $Z^{\prime} \gamma$ annihilation mode depends on both $m_{V}$ and $m_{Z^{\prime}}$.

$$
E_{\gamma} \simeq m_{V}\left(1-\frac{m_{Z^{\prime}}^{2}}{4 m_{V}^{2}}\right) \equiv m_{V}-\Delta E_{\gamma}
$$

where $\Delta E_{\gamma} \equiv \frac{m_{Z^{\prime}}^{2}}{4 m_{V}}$. If the $Z^{\prime} \gamma$ mode is kinematically allowed, namely for $m_{Z^{\prime}} \lesssim 2 m_{V}$, we have a chance to observe the double-peak gamma-ray spectrum at $E_{\gamma} \simeq m_{V}-\Delta E_{\gamma}$ and $E_{\gamma} \simeq m_{V}$. To distinguish between these two peaks, $\frac{\Delta E_{\gamma}}{m_{V}}$ should be larger than the instrumental energy resolution. In the H.E.S.S. experiment, the energy resolution is about $10 \%$ for $m_{\mathrm{DM}} \gtrsim 300 \mathrm{GeV}$. Our interesting region is

$$
1.02 \lesssim \frac{m_{Z^{\prime}}}{m_{V}}<2,
$$

to discuss the signal discrimination. The lower and upper values come from the $g_{0}$ perturbative unitarity and the kinematical suppression of the $Z^{\prime} \gamma$ annihilation mode, respectively. If we focus on this region, the condition $\frac{\Delta E_{\gamma}}{m_{V}} \gtrsim 0.1$ is always satisfied. Therefore, we can discriminate the gamma-ray peak originated from the $\gamma \gamma / Z \gamma$ modes and the peak from the $Z^{\prime} \gamma$ mode. This double-peak gamma-ray spectrum is an outstanding feature of our DM model, and we can read out the values of $m_{V}$ and $m_{Z^{\prime}}$ from this double-peak spectrum. ${ }^{7}$

We define the "line cross section", which contributes to the gamma-ray line signal. We introduce two types of line cross sections that predict the different final photon energy.

$$
\begin{aligned}
\left\langle\sigma v_{\text {rel }}\right\rangle_{\gamma \gamma, Z \gamma}^{\text {line }} & =\left\langle\sigma v_{\text {rel }}\right\rangle_{\gamma \gamma}+\frac{1}{2}\left\langle\sigma v_{\text {rel }}\right\rangle_{Z \gamma} & & \text { Energy peak: } E_{\gamma}=m_{V}, \\
\left\langle\sigma v_{\text {rel }}\right\rangle_{Z^{\prime} \gamma}^{\text {line }} & =\frac{1}{2}\left\langle\sigma v_{\text {rel }}\right\rangle_{Z^{\prime} \gamma} & & \text { Energy peak: } E_{\gamma}=m_{V}\left(1-\frac{m_{Z^{\prime}}^{2}}{4 m_{V}^{2}}\right) .
\end{aligned}
$$

We derive the excluded region by using the experimental bound shown in figure 6 of ref. [32]. In their analysis, all the final state particles are assumed to be massless, and thus we can directly compare these constraints with $\left\langle\sigma v_{\text {rel }}\right\rangle_{\gamma \gamma, Z \gamma}^{\text {line }}$. We can also derive the constraint on $\left\langle\sigma v_{\text {rel }}\right\rangle_{Z^{\prime} \gamma}^{\text {line }}$ by noting that the horizontal axis of figure 6 in ref. [32] corresponds to $E_{\gamma}$. These constraints are derived by assuming the three cuspy DM density profiles as defined below.

- Einasto profile [33]/Einasto2 profile [34]

$$
\rho_{\text {Einasto }}(r) \equiv \rho_{s} \exp \left[-\frac{2}{\alpha_{s}}\left(\left(\frac{r}{r_{s}}\right)^{\alpha_{s}}-1\right)\right] .
$$

\footnotetext{
${ }^{7}$ Similar signals are predicted in the context of the extra-dimensional models. See ref. [33] for the discussion in the model with six-dimensions.
} 


\begin{tabular}{|c|ccc|}
\hline Profiles & Einasto [33] & NFW [35] & Einasto2 [34] \\
\hline$\rho_{\mathrm{s}}\left[\mathrm{GeV} \mathrm{cm}{ }^{-3}\right]$ & 0.079 & 0.307 & 0.033 \\
$r_{\mathrm{s}}[\mathrm{kpc}]$ & 20.0 & 21.0 & 28.4 \\
$\alpha_{\mathrm{s}}$ & 0.17 & - & 0.17 \\
\hline
\end{tabular}

Table 2. The cuspy DM density profiles used in ref. [32].

- Navarro-Frenk-White (NFW) profile [35]

$$
\rho_{\mathrm{NFW}}(r) \equiv \rho_{s}\left(\frac{r}{r_{s}}\left(1+\frac{r}{r_{s}}\right)^{2}\right)^{-1} .
$$

In table. 2, we summarize the parameters for the cuspy DM density profiles used in ref. [32]. Another choice is the cored DM density profile. The core radius, $r_{c}$, depends on the model of baryonic physics, and cores extending to $r_{c} \sim 5 \mathrm{kpc}$ can potentially be obtained [36]. The cored Einasto profile is defined as shown below.

$$
\rho(r)= \begin{cases}\rho_{\text {Einasto }}(r) & \text { for } r>r_{c}, \\ \rho_{\text {Einasto }}\left(r_{c}\right) & \text { for } r<r_{c}\end{cases}
$$

where $\rho_{\text {Einasto }}(r)$ is defined in eq. (4.5). For the cored profile, $\rho_{s}$ is chosen to realize the value of the local DM density. We show the current excluded region with the cusped DM density profiles assumed in the analysis of the H.E.S.S. collaboration [32]. The sensitivity of the H.E.S.S. experiment is studied for the cored profiles in ref. [37], which is focusing on the pure Wino DM search. ${ }^{8}$ From this study, the upper bound on the line cross section will be weakened by a factor of $\mathcal{O}(10-100)$ if we use the cored DM density profile.

\subsection{Constraint from gamma-ray line signatures}

We compare the predicted line cross section between our spin-1 DM and the Wino system. In figure 1, we show the predicted value of $\left\langle\sigma v_{\text {rel }}\right\rangle_{\gamma \gamma, Z \gamma}^{\text {line }}$ and experimental upper bounds. The solid (dashed) black curve shows the line cross section including Sommerfeld enhancement for our spin-1 DM (the pure Wino DM). The green region with solid boundary is the excluded region by the H.E.S.S. observation [32] for the Einasto profile [33]. We also show green dashed and green dotted curves as the upper limits on the cross section for the cusped NFW profile [35] and the Einasto2 profile [34], respectively. Since our spin-1 DM and the Wino DM have the $\mathrm{SU}(2)_{L}$ triplet features, the Sommerfeld resonance structures are almost the same. The line cross section for the spin-1 DM is larger than that for the Wino DM by $\frac{38}{9}$, and thus we obtain more severe constraints on the spin-1 DM. We find the following excluded regions for our spin-1 DM depending on the DM density profiles. ${ }^{9}$

\footnotetext{
${ }^{8}$ See the left panel of figure 7 in ref. [37].

${ }^{9}$ If we take $m_{h^{\prime}} \simeq m_{V}$, the parameter region in figure 2 may be constrained by the perturbative unitarity bounds on the scalar couplings, which is studied in our previous collaboration [15]. We can evade these unitarity bounds by taking sufficiently small values of $\phi_{h}$ and $m_{h^{\prime}}$.
} 


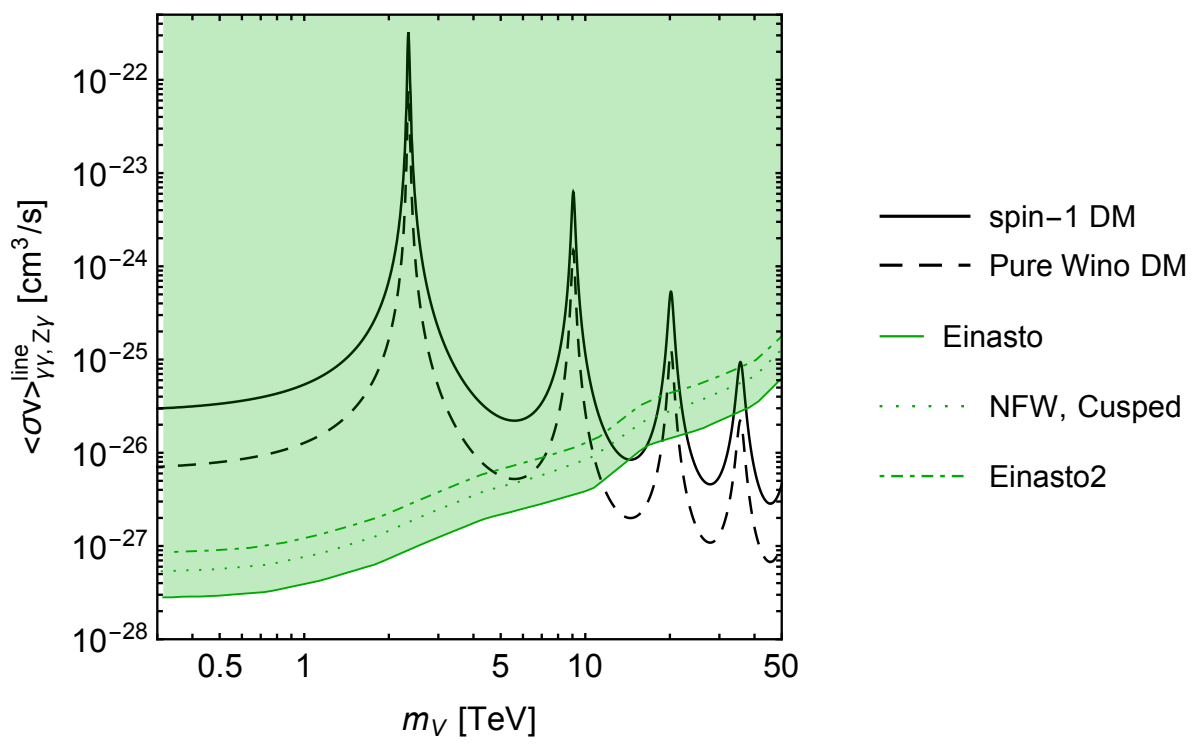

Figure 1. The comparison of the line cross sections between our spin-1 DM and the Wino DM. The solid (dashed) black curve shows $\left\langle\sigma v_{\text {rel }}\right\rangle_{\gamma \gamma, Z \gamma}^{\text {line }}$ for our model (pure Wino). The green shaded region shows the constraint from the H.E.S.S. observation in the Galactic Center region [32] for the Einasto profile [33]. The green dotted curve and dashed curve show the upper limit for the NFW profile [35] and Einasto2 profile [34], respectively. The upper bound is expected to be weakened by a factor of $\mathcal{O}(10-100)$ for the cored DM density profile.

- Einasto profile

$$
\begin{aligned}
& 300 \mathrm{GeV} \lesssim m_{V} \lesssim 14.4 \mathrm{TeV} \quad \text { (Excluded region 1) } \\
&\left.16.5 \mathrm{TeV} \lesssim m_{V} \lesssim 22.9 \mathrm{TeV} \quad \text { (Excluded region } 2\right), \\
&\left.33.8 \mathrm{TeV} \lesssim m_{V} \lesssim 37.5 \mathrm{TeV} \quad \text { (Excluded region } 3\right) .
\end{aligned}
$$

- Cusped NFW profile

$$
\begin{aligned}
& 300 \mathrm{GeV} \lesssim m_{V} \lesssim 12.5 \mathrm{TeV} \quad \text { (Excluded region 1) } \\
&\left.17.9 \mathrm{TeV} \lesssim m_{V} \lesssim 22.2 \mathrm{TeV} \quad \text { (Excluded region } 2\right), \\
&\left.34.8 \mathrm{TeV} \lesssim m_{V} \lesssim 36.7 \mathrm{TeV} \quad \text { (Excluded region } 3\right) .
\end{aligned}
$$

- Einasto2 profile

$$
\begin{aligned}
& 300 \mathrm{GeV} \lesssim m_{V} \lesssim 11.7 \mathrm{TeV} \quad \text { (Excluded region 1) } \\
& 18.6 \mathrm{TeV} \lesssim m_{V} \lesssim 21.6 \mathrm{TeV} \quad \text { (Excluded region 2) } \\
& 35.0 \mathrm{TeV} \lesssim m_{V} \lesssim 36.1 \mathrm{TeV} \quad \text { (Excluded region 3). }
\end{aligned}
$$

The lower value of $300 \mathrm{GeV}$ comes from the limitation for the energy resolution in the H.E.S.S experiment. 


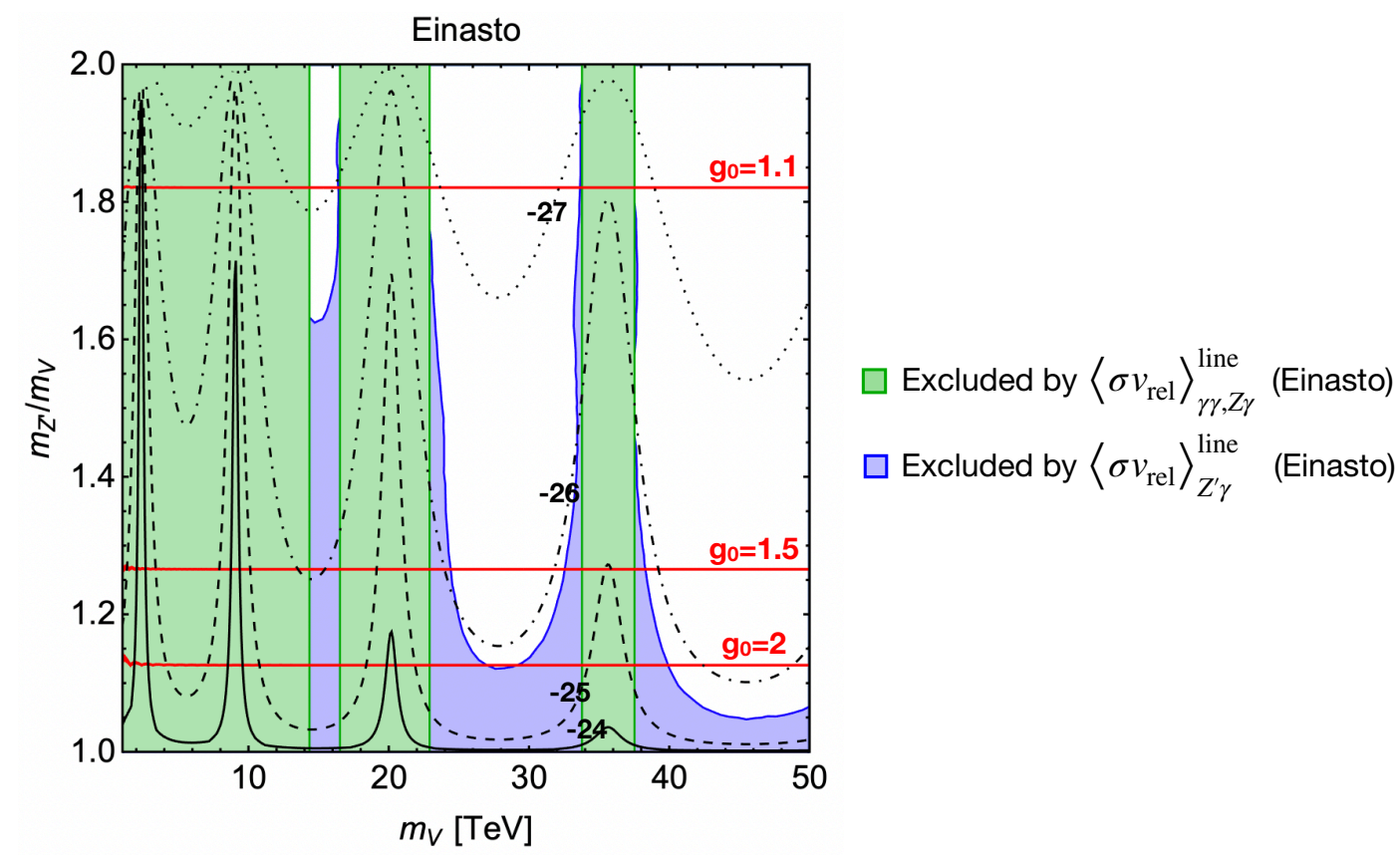

Figure 2. The current bound on the line cross section by the H.E.S.S. observation for the Einasto profile [33]. The green regions are excluded by $\left\langle\sigma v_{\text {rel }}\right\rangle_{\gamma \gamma, Z \gamma}^{\text {line }}$. The blue regions are excluded by $\left\langle\sigma v_{\text {rel }}\right\rangle_{Z^{\prime} \gamma}^{\text {line }}$. The black solid, dashed, dot-dashed, and dotted contours show the predicted value of $\left\langle\sigma v_{\text {rel }}\right\rangle_{Z^{\prime} \gamma}^{\text {line }}$ for $10^{-24}, 10^{-25}, 10^{-26}$, and $10^{-27}$ in the unit of $\mathrm{cm}^{3} / \mathrm{s}$, respectively. The red solid lines show the $g_{0}$ contours.

In figure 2, we show the current bound on the line cross section by the H.E.S.S. observation for the Einasto DM density profiles [33] focusing on the region in eq. (4.2). The green regions are excluded by $\left\langle\sigma v_{\text {rel }}\right\rangle_{\gamma \gamma, Z \gamma}^{\text {line }}$ shown in eqs. (4.8)-(4.10). These green regions are extended for $\frac{m_{Z^{\prime}}}{m_{V}} \geq 2$ where the annihilation into $Z^{\prime} \gamma$ is forbidden kinematically. The blue region is excluded by $\left\langle\sigma v_{\text {rel }}\right\rangle_{Z^{\prime} \gamma}^{\text {line }}$. The black solid, dashed, dot-dashed, and dotted contours show the predicted value of $\left\langle\sigma v_{\text {rel }}\right\rangle_{Z^{\prime} \gamma}^{\text {line }}$ for $10^{-24}, 10^{-25}, 10^{-26}$, and $10^{-27}$ in the unit of $\mathrm{cm}^{3} / \mathrm{s}$, respectively. The red solid lines show the $g_{0}$ contours. In figure 3 , we show the constraints for the NFW [35], and Einasto2 DM density profiles [34]. The derived constraints depend on the DM density profiles. The blue excluded regions from $\left\langle\sigma v_{\text {rel }}\right\rangle_{Z^{\prime} \gamma}^{\text {line }}$ give stronger constraints for $m_{Z^{\prime}} \simeq m_{V}$. This is because we have the enhancement factor in the coupling of $Z^{\prime}, g_{Z^{\prime}}$, defined in eq. (2.47). Note that $g_{0}$ gets larger in the same region, and thus we expect relatively large higher-order correction for our perturbative calculations.

We have a further chance to explore the parameter region in the upcoming Cherenkov Telescope Array (CTA) [38, 39]. The prospect sensitivity for the line gamma-ray signals is studied by ref. [40] for the Wino and Higgsino DM. In figure 4, we show the current bound and the future sensitivity expected in the CTA. The green region is excluded by the H.E.S.S. observation for the cusped Einasto2 profile shown in the lower panel of figure 3. We use the prospect bound derived in ref. [40] to show the CTA sensitivity. The orange region with the dashed boundary shows the most conservative sensitivity assuming the core 


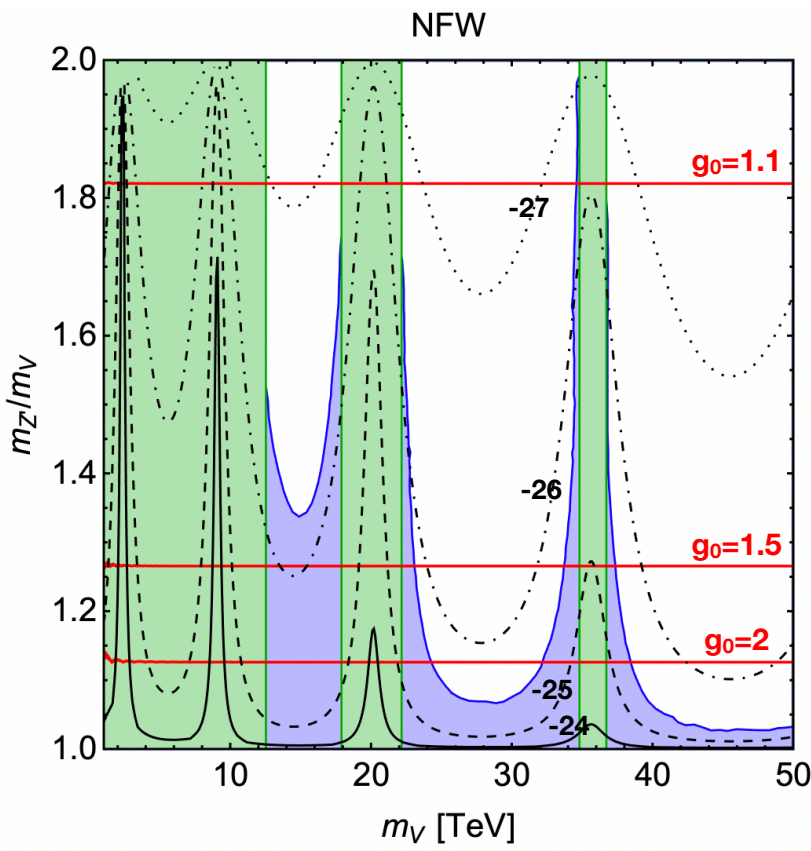

$\square$ Excluded by $\left\langle\sigma v_{\text {rel }}\right\rangle_{\gamma \gamma, Z \gamma}^{\text {line }}$ (NFW)
$\square$ Excluded by $\left\langle\sigma v_{\text {rel }}\right\rangle_{Z^{\prime} \gamma}^{\text {line }}(\mathrm{NFW})$

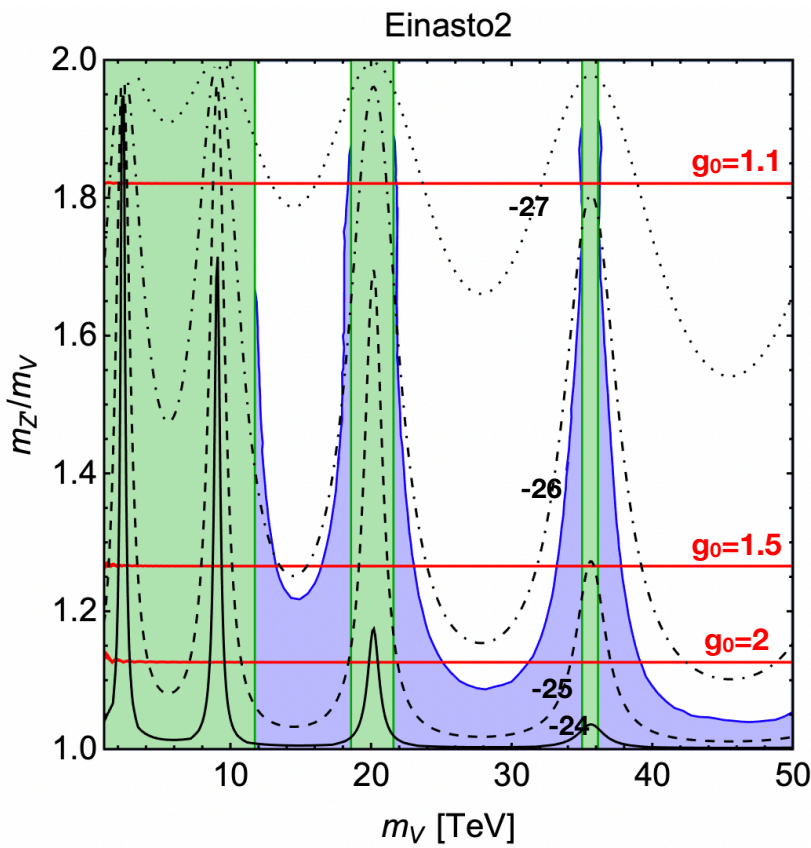

$\square$ Excluded by $\left\langle\sigma v_{\text {rel }}\right\rangle_{\gamma \gamma, Z \gamma}^{\text {line }}$ (Einasto2)

Excluded by $\left\langle\sigma v_{\text {rel }}\right\rangle_{Z^{\prime} \gamma}^{\text {line }} \quad$ (Einasto2)

Figure 3. The constraints on the line cross section for the different DM density profiles. The upper and lower panels show the constraints for the NFW profile [35] and the Einasto2 profile [34], respectively. The explanations for each plot are given in the caption of figure 2. 


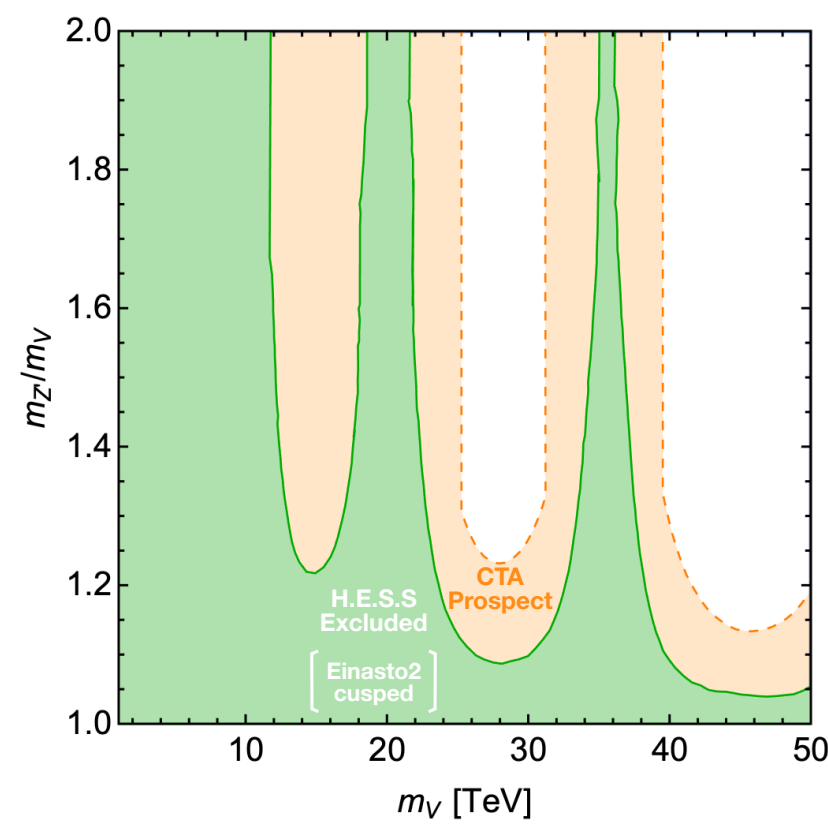

H.E.S.S Excluded (Einasto2, cusped)

CTA Prospect (Einasto, cored $r_{c}=5 \mathrm{kpc}$ )

Figure 4. The current bound from the H.E.S.S. observation and the prospect in the CTA experiment. The green region is excluded by the H.E.S.S. collaboration for the Einasto2 profile [34] shown in the lower panel of figure 3. The orange region with the dashed boundary is the prospect sensitivity in the CTA experiment for the cored Einasto profile with $r_{c}=5 \mathrm{kpc}$, which is the most conservative one [40]. If we take $r_{c} \lesssim 2 \mathrm{kpc}$, the prospect sensitivity will cover the whole region of this figure.

radius $r_{c}=5 \mathrm{kpc}$, and we will obtain $m_{V} \gtrsim 25.3 \mathrm{TeV}$ as the prospect bound. The whole mass range in figure 4 will be covered if we take $r_{c} \lesssim 2 \mathrm{kpc}$.

\section{Conclusions}

In this paper, we study the gamma-ray line signatures from the electroweakly interacting non-abelian vector DM model. We derive the two-body effective action for the spin-1 DM in the NR limit. Since our DM has the $\mathrm{SU}(2)_{L}$ triplet-like features, the Sommerfeld enhancement factor is almost the same as that for the pure Wino DM. The predicted annihilation cross sections into $\gamma \gamma / Z \gamma$ are larger by $\frac{38}{9}$ than those of the Wino. This is because our spin-1 DM pair has the additional partial wave contributions with the total spin angular momentum $J=2$. Therefore, the gamma-ray line signals provide stronger constraints on our spin-1 DM compared with the Wino DM.

We show the constraints on the line cross section for the $\gamma \gamma, Z \gamma$, and $Z^{\prime} \gamma$ modes by the H.E.S.S. observation in the Galactic Center region. The photon from the $\gamma \gamma / Z \gamma$ modes and the $Z^{\prime} \gamma$ mode are separable through the final photon energy. Therefore, we may observe a double-peak in the gamma-ray energy spectrum, which provides a unique signature of our spin-1 DM. The constraints strongly depend on the DM density profiles, and we obtain $m_{V} \gtrsim 14.4 \mathrm{TeV}(11.7 \mathrm{TeV})$ for the Einasto (Einasto2) profile. The annihilation into $Z^{\prime} \gamma$ also gives a strong constraint for $m_{V} \simeq m_{Z^{\prime}}$ where couplings between DM and $Z^{\prime}$ are enhanced. 

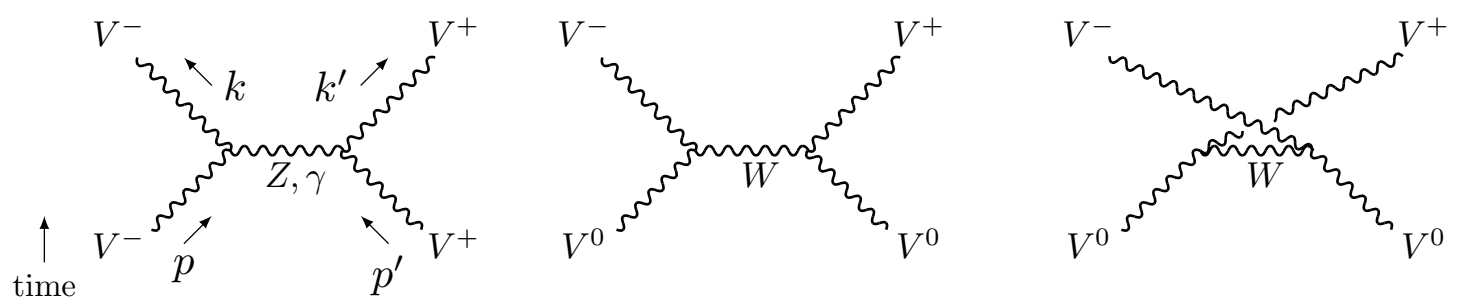

Figure 5. The relevant tree-level diagrams to derive the real part of the potential. The diagrams for $V^{-} V^{+} \rightarrow V^{0} V^{0}$ mode are implicit.

We also show the future sensitivity in the CTA experiment. We can probe $m_{V} \gtrsim 25.3 \mathrm{TeV}$ even if we take the conservative cored DM density profile with the core radius of $5 \mathrm{kpc}$.

We have possible extensions of our study. We can derive a more robust constraint on our model by studying the continuous gamma-ray search in observations of dwarf spheroidal galaxies by the Fermi-LAT experiment [41, 42]. We also expect a viable constraint from the antiproton observations as studied for the pure Wino DM [43]. We need to follow the decay chain including the decay of the $W^{\prime} / Z^{\prime}$ bosons in our model. Although we expect the strongest bound comes from the gamma-ray line signatures studied in this paper, we suffer a large uncertainty in the DM density profiles. Therefore, it is worth evaluating the more robust constraints from the other channels. We also expect the Sommerfeld enhancement affects the calculations of the thermal relic abundance, which we need to derive the effective action for the electrically charged two-body states [31, 44]. These studies are completed in our future collaborations.

\section{Acknowledgments}

JH thanks Seong Chan Park for useful discussions. This work was supported by JSPS KAKENHI Grant Numbers 19H04615 and 21K03549 (TA), and JSPS Grant-in-Aid for Scientific Research KAKENHI Grant Numbers JP20J12392 (MF), JP20H01895 (JH), and JP21K03572 (JH). The work is also supported by JSPS Core-to-Core Program (grant number:JPJSCCA20200002). The work of JH was supported by Grant-in-Aid for Scientific research from the Ministry of Education, Science, Sports, and Culture (MEXT), Japan (Grant Numbers 16H06492). The work of JH was also supported by World Premier International Research Center Initiative (WPI Initiative), MEXT, Japan.

\section{A Derivation of effective action}

We give the derivation of the effective action shown in section 3 .

\section{A.1 Real part of potential}

The real part of the potential is derived to realize the NR amplitudes of the $V$-particles. The leading-order contributions are induced from the diagrams shown in figure 5. Compared with the leading-order contributions, the Higgs exchange contributions are suppressed by 
small $\phi_{h}$. The $W^{\prime}$ and $Z^{\prime}$ exchange contributions are exponentially suppressed by $m_{W^{\prime}}$ and $m_{Z^{\prime}}$. The contributions from the vector quadratic couplings are suppressed by $\frac{1}{m_{V}^{2}}$. In the NR limit, we obtain the following amplitude at the leading-order.

$$
\begin{gathered}
i \mathcal{M}_{Z, \gamma} \simeq i 4 m_{V}^{2}\left(\frac{e^{2}}{\left|\boldsymbol{p}-\boldsymbol{p}^{\prime}\right|^{2}}+\frac{g_{W}^{2} c_{W}^{2}}{\left|\boldsymbol{p}-\boldsymbol{p}^{\prime}\right|^{2}+m_{Z}^{2}}\right) \epsilon_{i}(p) \epsilon_{i}^{*}(k) \epsilon_{j}\left(p^{\prime}\right) \epsilon_{j}^{*}\left(k^{\prime}\right), \\
i \mathcal{M}_{W} \simeq i 4 m_{V}^{2}\left(\frac{g_{W}^{2}}{\left|\boldsymbol{p}-\boldsymbol{p}^{\prime}\right|^{2}+m_{W}^{2}} \epsilon_{i}(p) \epsilon_{i}^{*}(k) \epsilon_{j}\left(p^{\prime}\right) \epsilon_{j}^{*}\left(k^{\prime}\right)\right. \\
\left.+\frac{g_{W}^{2}}{\left|\boldsymbol{p}-\boldsymbol{k}^{\prime}\right|^{2}+m_{W}^{2}} \epsilon_{i}(p) \epsilon_{i}^{*}\left(k^{\prime}\right) \epsilon_{j}\left(p^{\prime}\right) \epsilon_{j}^{*}(k)\right)
\end{gathered}
$$

where $\mathcal{M}_{Z, \gamma}$ and $\mathcal{M}_{W}$ corresponds to the neutral and charged boson exchange processes, respectively. The labels of the polarization are implicit. We obtain the effective action composed of the NR $V$-particle operators, which are defined in eqs. (3.12)-(3.14), to realize the above amplitudes.

$$
\begin{array}{rl}
S_{\mathrm{eff}}=\int d^{4} & R d^{3} r \frac{\alpha_{2} s_{W}^{2}+\alpha_{2} c_{W}^{2} e^{-m_{Z^{r}}}}{r} \\
& \times\left[\mathcal{B}_{i}^{\dagger}\left(R^{0}, \boldsymbol{R}+\boldsymbol{r} / 2\right) \mathcal{B}_{i}\left(R^{0}, \boldsymbol{R}+\boldsymbol{r} / 2\right)\right]\left[\mathcal{D}_{j}^{\dagger}\left(R^{0}, \boldsymbol{R}-\boldsymbol{r} / 2\right) \mathcal{D}_{j}\left(R^{0}, \boldsymbol{R}-\boldsymbol{r} / 2\right)\right] \\
+ & \left\{\int d^{4} R d^{3} r \frac{\alpha_{2} e^{-m_{W} r}}{r}\right. \\
& \times\left[\mathcal{A}_{i}^{\dagger}\left(R^{0}, \boldsymbol{R}+\boldsymbol{r} / 2\right) \mathcal{B}_{i}\left(R^{0}, \boldsymbol{R}+\boldsymbol{r} / 2\right)\right]\left[\mathcal{A}_{j}^{\dagger}\left(R^{0}, \boldsymbol{R}-\boldsymbol{r} / 2\right) \mathcal{D}_{j}\left(R^{0}, \boldsymbol{R}-\boldsymbol{r} / 2\right)\right] \\
+ & \text { h.c. }\} .
\end{array}
$$

To express the action in the decomposed form into each partial wave mode, we reform the vector indices $(i, j)$ by using the Fierz identity for Grassmann-even operators.

$$
\delta_{i j} \delta_{k \ell}=\sum_{J, J_{z}}(-1)^{J} S_{i \ell}^{J, J_{z}} S_{k j}^{J, J_{z} *} \quad(i, j, k, \ell=1,2,3),
$$

where $S_{i j}^{J, J_{z}}$ is defined in eqs. (3.24)-(3.26). After this decomposition, we can express the action in terms of the two-body states defined in eqs. (3.21)-(3.22) and read out $\hat{V}$ as given in eq. (3.29).

\section{A.2 Imaginary part of potential}

\section{A.2.1 Matching procedure}

To derive the imaginary part of the potential, $\hat{\Gamma}^{J}$, we perform the operator matching between the two-body field operators and the calculations of the one-loop amplitudes of the $V$-particles. Since we focus on the annihilation modes into the neutral vectors, the $(1,1)$-component of $\hat{\Gamma}^{J}$ only has the nonzero value. Therefore, we can use the optical theorem to calculate the imaginary part of the one-loop amplitude.

$$
\left.\operatorname{Im} \mathcal{M}\right|_{V^{-} V^{+} \rightarrow X X^{\prime} \rightarrow V^{-} V^{+}} ^{J}=2 m_{V}^{2}\left\langle\sigma v_{\text {rel }}\right\rangle_{V^{-} V^{+} \rightarrow X X^{\prime}}^{J} \quad\left(X X^{\prime}=\gamma \gamma, Z \gamma, Z^{\prime} \gamma\right),
$$


(1) 4-coupling diagram

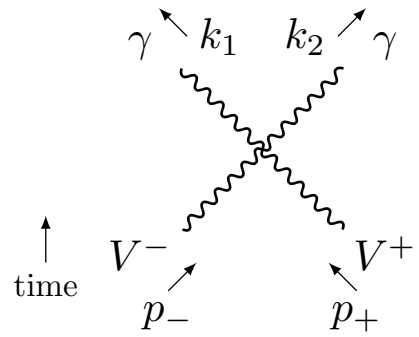

(2) t-channel diagram

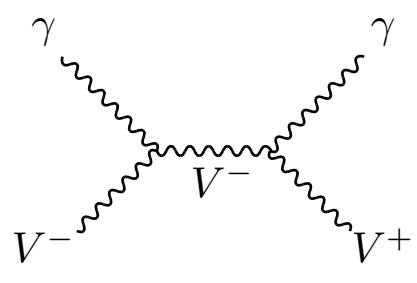

(3) u-channel diagram

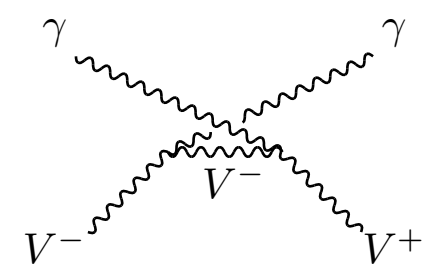

Figure 6. The tree-level diagrams which contribute to $V^{-} V^{+} \rightarrow \gamma \gamma$ annihilation.

where the left-hand side denotes the imaginary part of the forward scattering amplitude for $V^{-} V^{+} \rightarrow X X^{\prime} \rightarrow V^{-} V^{+}$with $J$ which is expressed by $\left(\hat{\Gamma}_{X X^{\prime}}^{J}\right)_{11}$. In the right-hand side, $\left\langle\sigma v_{\text {rel }}\right\rangle_{V^{-} V^{+} \rightarrow X X^{\prime}}^{J}$ denotes the partial wave annihilation cross section of $V^{-} V^{+} \rightarrow X X^{\prime}$ for the initial state with $J$. In the evaluation of the annihilation cross section, we only leave the leading-order terms in the NR limit and take the massless limit for all the SM particles in the final states while we leave the masses of $Z^{\prime}$. We derive $\hat{\Gamma}^{J}$ through the above procedure as summarized in eqs. (3.30)-(3.35). In the succeeding section, we show how to determine $\hat{\Gamma}_{\gamma \gamma}^{J}$ as a demonstration.

\section{A.2.2 Derivation of $\hat{\Gamma}_{\gamma \gamma}^{J}$}

We show the derivation of $\hat{\Gamma}_{\gamma \gamma}^{J}$ as a concrete example. We can focus on the nonzero component, $\left(\hat{\Gamma}_{\gamma \gamma}^{J}\right)_{11}$. First, we calculate the velocity-weighted annihilation cross section for $V^{-} V^{+} \rightarrow \gamma \gamma$. The tree-level diagrams in the unitarity gauge are shown in figure 6 . The amplitudes that correspond to each diagram are obtained as follows.

$$
\begin{aligned}
i \mathcal{M}_{4}= & i e^{2}\left(g^{\mu \rho} g^{\nu \sigma}+g^{\nu \rho} g^{\mu \sigma}-2 g^{\mu \nu} g^{\rho \sigma}\right) \epsilon_{\sigma}\left(p_{-}\right) \epsilon_{\rho}\left(p_{+}\right) \epsilon_{\mu}^{*}\left(k_{1}\right) \epsilon_{\nu}^{*}\left(k_{2}\right) \\
i \mathcal{M}_{t}= & i e\left[\left(p_{-}+q\right)^{\mu} g^{\alpha \sigma}+\left(-p_{-}-k_{1}\right)^{\alpha} g^{\mu \sigma}+\left(k_{1}-q\right)^{\sigma} g^{\mu \alpha}\right] \frac{-i}{q^{2}-m_{V}^{2}}\left(g_{\alpha \beta}-\frac{q_{\alpha} q_{\beta}}{m_{V}^{2}}\right) \\
& \times i e\left[\left(q-p_{+}\right)^{\nu} g^{\beta \rho}+\left(-q-k_{2}\right)^{\rho} g^{\beta \nu}+\left(k_{2}+p_{+}\right)^{\beta} g^{\rho \nu}\right] \epsilon_{\sigma}\left(p_{-}\right) \epsilon_{\rho}\left(p_{+}\right) \epsilon_{\mu}^{*}\left(k_{1}\right) \epsilon_{\nu}^{*}\left(k_{2}\right), \\
i \mathcal{M}_{u}= & i e\left[\left(p_{-}+q^{\prime}\right)^{\nu} g^{\alpha \sigma}+\left(-p_{-}-k_{2}\right)^{\alpha} g^{\nu \sigma}+\left(k_{2}-q^{\prime}\right)^{\sigma} g^{\nu \alpha}\right] \frac{-i}{q^{\prime 2}-m_{V}^{2}}\left(g_{\alpha \beta}-\frac{q_{\alpha}^{\prime} q_{\beta}^{\prime}}{m_{V}^{2}}\right) \\
& \times i e\left[\left(q^{\prime}-p_{+}\right)^{\mu} g^{\beta \rho}+\left(-q^{\prime}-k_{1}\right)^{\rho} g^{\beta \mu}+\left(k_{1}+p_{+}\right)^{\beta} g^{\rho \mu}\right] \epsilon_{\sigma}\left(p_{-}\right) \epsilon_{\rho}\left(p_{+}\right) \epsilon_{\mu}^{*}\left(k_{1}\right) \epsilon_{\nu}^{*}\left(k_{2}\right),
\end{aligned}
$$

where $q \equiv p_{-}-k_{1}$ and $q^{\prime} \equiv p_{-}-k_{2}$.

In the NR limit for the initial $V$-particles, the zeroth components of the polarization vectors are sub-leading, and we focus on the spatial component, $\epsilon_{i}\left(p_{-}\right) \epsilon_{j}\left(p_{+}\right)$. We decompose the amplitude into each partial wave contribution by replacing the initial state polarization vectors with $S_{i j}^{J, J_{z}}$ defined in eqs. (3.24)-(3.26).

$$
\epsilon_{i}\left(p_{-}\right) \epsilon_{j}\left(p_{+}\right) \rightarrow S_{i j}^{J, J_{z}}
$$


Note that $S_{i j}^{J, J_{z}}$ is symmetric matrices for $J=0,2$ and anti-symmetric for $J=1$. Thanks to these properties, the amplitudes in the center-of-mass frame are obtained in the decomposed form, $\mathcal{M}^{J, J_{z}}$.

$$
\begin{aligned}
\mathcal{M}^{0,0} & =2 e^{2}\left[4 \epsilon_{i}^{*}\left(k_{1}\right) S_{i j}^{0,0} \epsilon_{j}^{*}\left(k_{2}\right)-\epsilon_{i}^{*}\left(k_{1}\right) \epsilon_{i}^{*}\left(k_{2}\right)\left(S_{i i}^{0,0}-\frac{2}{m_{V}^{2}} k_{j} S_{j k}^{0,0} k_{k}\right)\right], \\
\mathcal{M}^{1, J_{z}} & =0 \\
\mathcal{M}^{2, J_{z}} & =2 e^{2}\left[4 \epsilon_{i}^{*}\left(k_{1}\right) S_{i j}^{2, J_{z}} \epsilon_{j}^{*}\left(k_{2}\right)+\epsilon_{i}^{*}\left(k_{1}\right) \epsilon_{i}^{*}\left(k_{2}\right) \frac{2}{m_{V}^{2}} k_{j} S_{j k}^{2, J_{z}} k_{k}\right],
\end{aligned}
$$

where $\left|J_{z}\right| \leq J$ and $k_{i}$ denotes the spatial components of $k_{1 \mu}$. We use $S_{i i}^{2, J_{z}}=0$ to obtain the above result. The polarization vectors for the photons are given by

$$
\epsilon_{i}^{ \pm}\left(k_{1}\right)=\frac{1}{\sqrt{2}}\left(\begin{array}{c}
\mp \cos \theta \\
-i \\
\pm \sin \theta
\end{array}\right), \quad \epsilon_{i}^{ \pm}\left(k_{2}\right)=\frac{1}{\sqrt{2}}\left(\begin{array}{c} 
\pm \cos \theta \\
-i \\
\mp \sin \theta
\end{array}\right),
$$

where $\theta$ is the scattering angle for the photon with its momentum $k_{1}$.

After taking the sum over the final state spins, the squared amplitudes can be expressed as follow.

$$
\begin{aligned}
& \sum_{\text {final }}\left|\mathcal{M}^{0,0}\right|^{2}=24 e^{4} \\
& \sum_{\text {final }}\left|\mathcal{M}^{1, J_{z}}\right|^{2}=0 \\
& \sum_{\text {final }}\left|\mathcal{M}^{2, J_{z}}\right|^{2}= \begin{cases}48 e^{4} \sin ^{4} \theta & \left(J_{z}=0, \pm 1\right), \\
16 e^{4} \sin ^{2} \theta(3+\cos 2 \theta) & \left(J_{z}= \pm\right) \\
8 e^{4}\left(1+6 \cos ^{2} \theta+\cos ^{4} \theta\right) & \left(J_{z}= \pm 2\right) .\end{cases}
\end{aligned}
$$

We obtain the cross section for each partial wave mode.

$$
\begin{aligned}
\left\langle\sigma v_{\text {rel }}\right\rangle_{V^{-} V^{+} \rightarrow \gamma \gamma}^{J=0} & =6 \frac{\pi \alpha_{2}^{2}}{m_{V}^{2}} s_{W}^{4}, \\
\left\langle\sigma v_{\text {rel }}\right\rangle_{V^{-} V^{+} \rightarrow \gamma \gamma}^{J=1} & =0, \\
\left\langle\sigma v_{\text {rel }}\right\rangle_{V^{-} V^{+} \rightarrow \gamma \gamma}^{J=2} & =\frac{32}{5} \frac{\pi \alpha_{2}^{2}}{m_{V}^{2}} s_{W}^{4} .
\end{aligned}
$$

The spin-averaged total cross section is obtained by adding up all the partial wave cross sections.

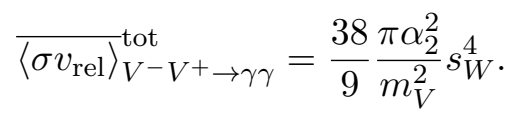


This cross section is larger than that of the Wino DM by a factor of $\frac{38}{9}$. For the $Z \gamma$ mode, we take the massless limit of the $Z$ boson, and the calculation procedure is the same. For the $Z^{\prime} \gamma$ mode, we do not neglect $m_{Z^{\prime}}$, and the longitudinal mode also contributes to the final result.

Next, we calculate the imaginary part of the forward scattering amplitude using the two-body effective action shown in eq. (3.19). Namely, we use the following coupling to calculate the $\gamma \gamma$ contribution to $\operatorname{Im} \mathcal{M}$.

$$
S_{\mathrm{eff}} \supset \sum_{J, J_{z}} i \frac{9}{2}\left(\hat{\Gamma}_{\gamma \gamma}^{J}\right)_{11} \int d^{4} R \phi_{C}^{J, J_{z} \dagger}(R, \mathbf{0}) \phi_{C}^{J, J_{z}}(R, \mathbf{0}) .
$$

Using this coupling, we obtain

$$
\left.\operatorname{Im} \mathcal{M}\right|_{V^{-} V^{+} \rightarrow \gamma \gamma \rightarrow V^{-} V^{+}} ^{J}=2 m_{V}^{2} \cdot 9\left(\hat{\Gamma}_{\gamma \gamma}^{J}\right)_{11} .
$$

Comparing with (A.5), we can determine $\left(\hat{\Gamma}_{\gamma \gamma}^{J}\right)_{11}$ as shown below.

$$
\left(\hat{\Gamma}_{\gamma \gamma}^{J}\right)_{11}=\frac{1}{9}\left\langle\sigma v_{\mathrm{rel}}\right\rangle_{V^{-} V^{+} \rightarrow \gamma \gamma}^{J}=\left\{\begin{array}{l}
\frac{2}{3} \frac{\pi \alpha_{2}^{2}}{m_{V}^{2}} s_{W}^{4}, \\
0, \\
\frac{32}{45} \frac{\pi \alpha_{2}^{2}}{m_{V}^{2}} s_{W}^{4} .
\end{array}\right.
$$

This result is summarized in eqs. (3.30)-(3.31). We can also determine $\hat{\Gamma}_{Z \gamma}^{J}$ and $\hat{\Gamma}_{Z^{\prime} \gamma}^{J}$ in the same procedure.

Open Access. This article is distributed under the terms of the Creative Commons Attribution License (CC-BY 4.0), which permits any use, distribution and reproduction in any medium, provided the original author(s) and source are credited.

\section{References}

[1] Planck collaboration, Planck 2018 results. VI. Cosmological parameters, Astron. Astrophys. 641 (2020) A6 [Erratum ibid. 652 (2021) C4] [arXiv: 1807.06209] [INSPIRE].

[2] B.W. Lee and S. Weinberg, Cosmological Lower Bound on Heavy Neutrino Masses, Phys. Rev. Lett. 39 (1977) 165 [INSPIRE].

[3] M. Cirelli, N. Fornengo and A. Strumia, Minimal dark matter, Nucl. Phys. B 753 (2006) 178 [hep-ph/0512090] [INSPIRE].

[4] M. Cirelli, A. Strumia and M. Tamburini, Cosmology and Astrophysics of Minimal Dark Matter, Nucl. Phys. B 787 (2007) 152 [arXiv:0706.4071] [INSPIRE].

[5] M. Cirelli and A. Strumia, Minimal Dark Matter: Model and results, New J. Phys. 11 (2009) 105005 [arXiv: 0903.3381] [INSPIRE].

[6] R. Amintaheri, Electroweak Dark Matter, arXiv:2105.11574 [INSPIRE].

[7] T. Moroi and L. Randall, Wino cold dark matter from anomaly mediated SUSY breaking, Nucl. Phys. B 570 (2000) 455 [hep-ph/9906527] [INSPIRE]. 
[8] N. Arkani-Hamed, A. Delgado and G.F. Giudice, The Well-tempered neutralino, Nucl. Phys. B 741 (2006) 108 [hep-ph/0601041] [INSPIRE].

[9] N. Nagata and S. Shirai, Higgsino Dark Matter in High-Scale Supersymmetry, JHEP 01 (2015) 029 [arXiv: 1410.4549] [InSPIRE].

[10] M. Beneke et al., Relic density of wino-like dark matter in the MSSM, JHEP 03 (2016) 119 [arXiv: 1601.04718] [INSPIRE].

[11] T. Flacke, A. Menon and D.J. Phalen, Non-minimal universal extra dimensions, Phys. Rev. D 79 (2009) 056009 [arXiv:0811.1598] [INSPIRE].

[12] N. Maru, N. Okada and S. Okada, $\mathrm{SU}(2)_{L}$ doublet vector dark matter from gauge-Higgs unification, Phys. Rev. D 98 (2018) 075021 [arXiv: 1803.01274] [INSPIRE].

[13] T. Flacke, D.W. Kang, K. Kong, G. Mohlabeng and S.C. Park, Electroweak Kaluza-Klein Dark Matter, JHEP 04 (2017) 041 [arXiv: 1702. 02949] [INSPIRE].

[14] A. Belyaev, G. Cacciapaglia, J. McKay, D. Marin and A.R. Zerwekh, Minimal Spin-one Isotriplet Dark Matter, Phys. Rev. D 99 (2019) 115003 [arXiv:1808.10464] [INSPIRE].

[15] T. Abe, M. Fujiwara, J. Hisano and K. Matsushita, A model of electroweakly interacting non-abelian vector dark matter, JHEP 07 (2020) 136 [arXiv:2004.00884] [INSPIRE].

[16] N. Arkani-Hamed, A.G. Cohen and H. Georgi, (De)constructing dimensions, Phys. Rev. Lett. 86 (2001) 4757 [hep-th/0104005] [INSPIRE].

[17] R. Coquereaux, Classical and quantum polyhedra: A Fusion graph algebra point of view, AIP Conf. Proc. 589 (2001) 181 [hep-th/0105239] [InSPIRE].

[18] J. Hisano, S. Matsumoto and M.M. Nojiri, Unitarity and higher order corrections in neutralino dark matter annihilation into two photons, Phys. Rev. D 67 (2003) 075014 [hep-ph/0212022] [INSPIRE].

[19] J. Hisano, S. Matsumoto and M.M. Nojiri, Explosive dark matter annihilation, Phys. Rev. Lett. 92 (2004) 031303 [hep-ph/0307216] [INSPIRE].

[20] J. Hisano, S. Matsumoto, M.M. Nojiri and O. Saito, Non-perturbative effect on dark matter annihilation and gamma ray signature from galactic center, Phys. Rev. D 71 (2005) 063528 [hep-ph/0412403] [INSPIRE].

[21] N. Arkani-Hamed, D.P. Finkbeiner, T.R. Slatyer and N. Weiner, A Theory of Dark Matter, Phys. Rev. D 79 (2009) 015014 [arXiv:0810.0713] [InSPIRE].

[22] K. Blum, R. Sato and T.R. Slatyer, Self-consistent Calculation of the Sommerfeld Enhancement, JCAP 06 (2016) 021 [arXiv: 1603.01383] [INSPIRE].

[23] T. Katayose, S. Matsumoto, S. Shirai and Y. Watanabe, Thermal real scalar triplet dark matter, JHEP 09 (2021) 044 [arXiv:2105.07650] [INSPIRE].

[24] K. Hally, H.E. Logan and T. Pilkington, Constraints on large scalar multiplets from perturbative unitarity, Phys. Rev. D 85 (2012) 095017 [arXiv: 1202.5073] [INSPIRE].

[25] T. Cohen, M. Lisanti, A. Pierce and T.R. Slatyer, Wino Dark Matter Under Siege, JCAP 10 (2013) 061 [arXiv:1307.4082] [INSPIRE].

[26] G. Ovanesyan, T.R. Slatyer and I.W. Stewart, Heavy Dark Matter Annihilation from Effective Field Theory, Phys. Rev. Lett. 114 (2015) 211302 [arXiv: 1409.8294] [INSPIRE].

[27] G. Ovanesyan, N.L. Rodd, T.R. Slatyer and I.W. Stewart, One-loop correction to heavy dark matter annihilation, Phys. Rev. D 95 (2017) 055001 [Erratum ibid. 100 (2019) 119901] [arXiv: 1612.04814] [INSPIRE]. 
[28] M. Beneke, A. Broggio, C. Hasner and M. Vollmann, Energetic $\gamma$-rays from TeV scale dark matter annihilation resummed, Phys. Lett. B $\mathbf{7 8 6}$ (2018) 347 [Erratum ibid. 810 (2020) 135831] [arXiv: 1805.07367] [INSPIRE].

[29] M. Beneke, A. Broggio, C. Hasner, K. Urban and M. Vollmann, Resummed photon spectrum from dark matter annihilation for intermediate and narrow energy resolution, JHEP 08 (2019) 103 [Erratum ibid. 07 (2020) 145] [arXiv: 1903.08702] [INSPIRE].

[30] M. Beneke, R. Szafron and K. Urban, Wino potential and Sommerfeld effect at NLO, Phys. Lett. B 800 (2020) 135112 [arXiv: 1909. 04584] [INSPIRE].

[31] M. Beneke, R. Szafron and K. Urban, Sommerfeld-corrected relic abundance of wino dark matter with NLO electroweak potentials, JHEP 02 (2021) 020 [arXiv: 2009. 00640] [INSPIRE].

[32] HESS collaboration, Search for $\gamma$-Ray Line Signals from Dark Matter Annihilations in the Inner Galactic Halo from 10 Years of Observations with H.E.S.S, Phys. Rev. Lett. 120 (2018) 201101 [arXiv:1805.05741] [INSPIRE].

[33] G. Bertone, C.B. Jackson, G. Shaughnessy, T.M.P. Tait and A. Vallinotto, The WIMP Forest: Indirect Detection of a Chiral Square, Phys. Rev. D 80 (2009) 023512 [arXiv: 0904.1442] [INSPIRE].

[34] M. Cirelli et al., PPPC 4 DM ID: A Poor Particle Physicist Cookbook for Dark Matter Indirect Detection, JCAP 03 (2011) 051 [Erratum ibid. 10 (2012) E01] [arXiv:1012.4515] [INSPIRE].

[35] J.F. Navarro, C.S. Frenk and S.D.M. White, A Universal density profile from hierarchical clustering, Astrophys. J. 490 (1997) 493 [astro-ph/9611107] [INSPIRE].

[36] P. Mollitor, E. Nezri and R. Teyssier, Baryonic and dark matter distribution in cosmological simulations of spiral galaxies, Mon. Not. Roy. Astron. Soc. 447 (2015) 1353 [arXiv: 1405.4318] [INSPIRE].

[37] L. Rinchiuso et al., Hunting for Heavy Winos in the Galactic Center, Phys. Rev. D 98 (2018) 123014 [arXiv:1808.04388] [INSPIRE].

[38] CTA Consortium collaboration, Dark Matter and Fundamental Physics with the Cherenkov Telescope Array, Astropart. Phys. 43 (2013) 189 [arXiv:1208.5356] [InSPIRE].

[39] CTA collaboration, Sensitivity of the Cherenkov Telescope Array to a dark matter signal from the Galactic centre, JCAP 01 (2021) 057 [arXiv: 2007.16129] [INSPIRE].

[40] L. Rinchiuso, O. Macias, E. Moulin, N.L. Rodd and T.R. Slatyer, Prospects for detecting heavy WIMP dark matter with the Cherenkov Telescope Array: The Wino and Higgsino, Phys. Rev. D 103 (2021) 023011 [arXiv: 2008. 00692] [INSPIRE].

[41] Fermi-LAT collaboration, Dark Matter Constraints from Observations of 25 Milky Way Satellite Galaxies with the Fermi Large Area Telescope, Phys. Rev. D 89 (2014) 042001 [arXiv: 1310.0828] [INSPIRE].

[42] S. Hoof, A. Geringer-Sameth and R. Trotta, A Global Analysis of Dark Matter Signals from 27 Dwarf Spheroidal Galaxies using 11 Years of Fermi-LAT Observations, JCAP 02 (2020) 012 [arXiv: 1812.06986] [INSPIRE].

[43] M. Ibe, S. Matsumoto, S. Shirai and T.T. Yanagida, Wino Dark Matter in light of the AMS-02 2015 Data, Phys. Rev. D 91 (2015) 111701 [arXiv:1504.05554] [INSPIRE].

[44] J. Hisano, S. Matsumoto, M. Nagai, O. Saito and M. Senami, Non-perturbative effect on thermal relic abundance of dark matter, Phys. Lett. B 646 (2007) 34 [hep-ph/0610249] [INSPIRE]. 